\title{
Optical Polarimetry for Fundamental Physics
}

\author{
Guido Zavattini ${ }^{1,2, *(D)}$ and Federico Della Valle ${ }^{3,4}$ (D) \\ 1 Dipartimento di Fisica e Scienze della Terra, Università di Ferrara, Via G. Saragat 1, I-44122 Ferrara, Italy \\ 2 INFN, Sezione di Ferrara, Via G. Saragat 1, I-44122 Ferrara, Italy \\ 3 Dipartimento di Scienze Fisiche, della Terra e dell'Ambiente, Università di Siena, Via Roma 56, \\ I-53100 Siena, Italy; federico.dellavalle@unisi.it \\ 4 INFN, Sezione di Pisa, Largo B. Pontecorvo 3, I-56127 Pisa, Italy \\ * Correspondence: guido.zavattini@unife.it
}

Citation: Zavattini, G.; Della Valle, F Optical Polarimetry for Fundamental Physics. Universe 2021, 7, 252. https://doi.org/10.3390/ universe7070252

Academic Editors: Janos Polonyi and Freya Blekman

Received: 1 June 2021

Accepted: 16 July 2021

Published: 20 July 2021

Publisher's Note: MDPI stays neutral with regard to jurisdictional claims in published maps and institutional affiliations.

Copyright: (c) 2021 by the authors. Licensee MDPI, Basel, Switzerland. This article is an open access article distributed under the terms and conditions of the Creative Commons Attribution (CC BY) license (https:// creativecommons.org/licenses/by/ $4.0 /)$.

\begin{abstract}
Sensitive magneto-optical polarimetry was proposed by E. Iacopini and E. Zavattini in 1979 to detect vacuum electrodynamic non-linearity, in particular Vacuum Magnetic Birefringence (VMB). This process is predicted in QED via the fluctuation of electron-positron virtual pairs but can also be due to hypothetical Axion-Like Particles (ALPs) and/or MilliCharged Particles (MCP). Today ALPs are considered a strong candidate for Dark Matter. Starting in 1992 the PVLAS collaboration, financed by INFN, Italy, attempted to measure VMB conceptually following the original 1979 scheme based on an optical cavity permeated by a time-dependent magnetic field and heterodyne detection. Two setups followed differing basically in the magnet: the first using a rotating superconducting $5.5 \mathrm{~T}$ dipole magnet at the Laboratori Nazionali di Legnaro, Legnaro, Italy and the second using two rotating permanent $2.5 \mathrm{~T}$ dipole magnets at the INFN section of Ferrara. At present PVLAS is the experiment which has set the best limit in VMB reaching a noise floor within a factor 7 of the predicted QED signal: $\Delta n^{(\mathrm{QED})}=2.5 \times 10^{-23} @ 2.5 \mathrm{~T}$. It was also shown that the noise floor was due to the optical cavity and a larger magnet is the only solution to increase the signal to noise ratio. The PVLAS experiment ended at the end of 2018. A new effort, VMB@CERN, which plans to use a spare LHC dipole magnet at CERN with a new modified optical scheme, is now being proposed. In this review, a detailed description of the PVLAS effort and the comprehension of its limits leading to a new proposal will be given.
\end{abstract}

Keywords: optical polarimetry; magneto-optic effects; optical tests of quantum electrodynamics

\section{Introduction}

From Maxwell's equations in vacuum the velocity of light is related to the magnetic permeability $\mu_{0}$ and vacuum permittivity $\varepsilon_{0}$ through the relation

$$
c=\frac{1}{\sqrt{\varepsilon_{0} \mu_{0}}} .
$$

Since 20 May 2019, the velocity of light in vacuum $c$ is defined to be $c=299,792,458 \mathrm{~m} / \mathrm{s}$ and $\varepsilon_{0}$ and $\mu_{0}$ are derived from the measurement of the fine structure constant $\alpha$ :

$$
\alpha=\frac{e^{2}}{4 \pi \varepsilon_{0} \hbar c}=\frac{e^{2} c \mu_{0}}{4 \pi \hbar}
$$

being $e$ and $\hbar$ also defined. Today's CODATA value is $\alpha^{-1}=137.035999206 \pm 0.000000011$. Due to the linearity of Maxwell's equations in vacuum the velocity of light in classical vacuum does not depend on the presence of other electromagnetic fields (photons, static fields).

The formulation of Einstein's energy-mass relation, Heisenberg's Uncertainty Principle and Dirac's relativistic equation of the electron opened the doors to vacuum fluctuations 
leading to nonlinear electrodynamics in vacuum such as light-by-light (LbL) elastic scattering and vacuum magnetic birefringence (VMB). These two closely related processes are shown in Figure 1 using today's Feynman diagrams. Interestingly, VMB is a macroscopic manifestation of a purely relativistic quantum mechanical effect resulting in a reduction of the speed of light in vacuum in the presence of an external field.
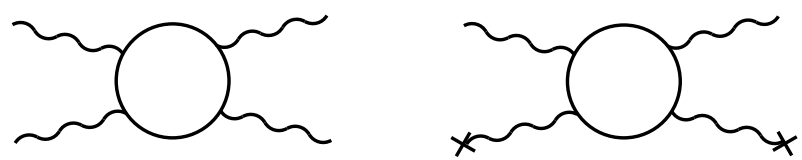

Figure 1. Lowest order nonlinear elementary processes in vacuum. (Left) light-by-light elastic scattering. (Right) vacuum magnetic birefringence.

QED is an extremely well tested theory but always in the presence of charged particles either in the initial and/or final states. The observation of VMB would represent a fundamental confirmation of QED with only low energy photons in the initial and final states.

In the present review paper included in a volume devoted to the Italian Research Infrastructure of relevance for astroparticle physics we will describe the acquired experience with the PVLAS experiment [1] along with its intrinsic limitations. The information presented in this paper is mostly contained in [1]. Here though we only present the most important issues to offer a less cumbersome reading. Moreover, this review also contains new experimental and conceptual developments in the field obtained during the last year. Indeed, a brief introduction will be given to a new optical polarimetric scheme [2] being proposed at CERN [3] and tested at the INFN section of Ferrara which overcomes the limitations of PVLAS. Hopefully, this new scheme will permit the first detection of this challenging, intriguing and fundamental process.

An effective Lagrangian density taking into account $e^{+} e^{-}$vacuum fluctuations was first written by H. Euler and B. Kockel in 1935 [4] and shortly after generalized by H. Euler, W. Heisenberg and V. S. Weisskopf [5-7] in 1936. The Lagrangian $\mathcal{L}_{\text {EK }}$ was later confirmed within the QED framework [8,9]. For electric and magnetic fields well below their critical values $\left(E \ll E_{\text {crit }}=m_{e}^{2} c^{3} / e \hbar=1.38 \times 10^{18} \mathrm{~V} / \mathrm{m}\right.$ and $\left.B \ll B_{\text {crit }}=m_{e}^{2} c^{2} / e \hbar=4.4 \times 10^{9} \mathrm{~T}\right)$ the resulting free field electromagnetic Lagrangian density was determined to be

$$
\mathcal{L}_{\mathrm{EK}}=\frac{1}{2 \mu_{0}}\left(\frac{E^{2}}{c^{2}}-B^{2}\right)+\frac{A_{e}}{\mu_{0}}\left[\left(\frac{E^{2}}{c^{2}}-B^{2}\right)^{2}+7\left(\frac{\vec{E}}{c} \cdot \vec{B}\right)^{2}\right]+\ldots
$$

where

$$
A_{e}=\frac{2}{45 \mu_{0}} \frac{\hbar^{3}}{m_{e}^{4} c^{5}} \alpha^{2}=1.32 \times 10^{-24} \mathrm{~T}^{-2} .
$$

It is apparent that $\mathcal{L}_{\mathrm{EK}}$, with terms to the 4 th power in the fields, admits 4 -field interactions. The parameter $A_{e}$ describes the entity of the nonlinear correction to the Classical Lagrangian and $\alpha=e^{2} /\left(4 \pi \epsilon_{0} \hbar c\right)$ is the fine structure constant. Allowing 4-field interactions, this Lagrangian leads to light-by-light (LbL) scattering [9,10], to a reduction of the velocity of light in the presence of an external field and to Vacuum Magnetic Birefringence (VMB) [11-16], namely a difference in the indices of refraction for light polarized parallel and perpendicular to an external magnetic field $\vec{B}_{\text {ext }}$. As of today $\mathcal{L}_{\mathrm{EK}}$ still needs direct experimental confirmation at energies below the electron mass. Evidence of LbL scattering of high energy virtual photons has been recently published by the ATLAS collaboration [17] and an indirect evidence of VMB has been published by Mignani et al. [18] (with some criticism [19]) as a result of observations of polarized light from an isolated neutron star.

The macroscopic properties of the quantum vacuum in the presence of an external field can be studied through the complex index of refraction $n_{c}=n+i \kappa$ where $n$ is the index of refraction and $\kappa$ is the extinction coefficient describing absorption. Furthermore, 
considering the propagation of a linearly polarized beam of light, if $n_{c}$ also depends on the polarization direction then $\Delta n$ is the birefringence and $\Delta \kappa$ is the dichroism.

By applying the Euler-Lagrange equations to $\mathcal{L}_{\text {EK }}$ one finds that electromagnetism in the presence of vacuum fluctuations is still described by Maxwell's equations but in a medium (in the absence of currents and charges)

$$
\begin{array}{ll}
\vec{\nabla} \cdot \vec{D}=0 & \vec{\nabla} \times \vec{E}=-\frac{\partial \vec{B}}{\partial t} \\
\vec{\nabla} \cdot \vec{B}=0 & \vec{\nabla} \times \vec{H}=\frac{\partial \vec{D}}{\partial t}
\end{array}
$$

where the relation between $\vec{H}$ and $\vec{B}$ and between $\vec{D}$ and $\vec{E}$ are given by

$$
\begin{aligned}
& \vec{H}=\frac{\vec{B}}{\mu_{0}}-\vec{M}=\frac{\vec{B}}{\mu_{0}}+\frac{A_{e}}{\mu_{0}}\left[4\left(\frac{E^{2}}{c^{2}}-B^{2}\right) \vec{B}-14 A_{e}\left(\frac{\vec{E}}{c} \cdot \vec{B}\right) \frac{\vec{E}}{c}\right] \\
& \vec{D}=\varepsilon_{0} \vec{E}+\vec{P}=\varepsilon_{0} \vec{E}+A_{e}\left[4\left(\frac{E^{2}}{c^{2}}-B^{2}\right) \varepsilon_{0} \vec{E}+14 \varepsilon_{0} A_{e}(\vec{E} \cdot \vec{B}) \vec{B}\right] .
\end{aligned}
$$

These relations result in a nonlinear anisotropic behavior of vacuum. The vectors $\vec{D}$ and $\vec{H}$ are no longer linear in the fields $\vec{E}$ and $\vec{B}$ and the indices of refraction for light polarized parallel and perpendicular to an external magnetic field are $n_{\|, \perp}>1$ and $n_{\|} \neq n_{\perp}$ resulting in VMB [12-15]. Considering a beam of linearly polarized light passing through a transverse magnetic field and substituting $\vec{E}=\vec{E}_{\text {light }}$ and $\vec{B}=\vec{B}_{\text {light }}+\vec{B}_{\text {ext }}$ one finds

$$
\begin{aligned}
& \vec{D}_{\text {light }}=\varepsilon_{0}\left[\vec{E}_{\text {light }}-4 A_{e} B_{\text {ext }}^{2} \vec{E}_{\text {light }}+14 A_{e}\left(\vec{E}_{\text {light }} \cdot \vec{B}_{\text {ext }}\right) \vec{B}_{\text {ext }}\right] \\
& \vec{H}_{\text {light }}=\frac{1}{\mu_{0}}\left[\vec{B}_{\text {light }}-4 A_{e} B_{\text {ext }}^{2} \vec{B}_{\text {light }}-8 A_{e}\left(\vec{B}_{\text {light }} \cdot \vec{B}_{\text {ext }}\right) \vec{B}_{\text {ext }}\right]
\end{aligned}
$$

from which one can determine $n=\sqrt{\varepsilon_{r} \mu_{r}}$ in the two cases:

$$
\left.\begin{array}{l}
n_{\|}=1+7 A_{e} B_{\mathrm{ext}}^{2} \\
n_{\perp}=1+4 A_{e} B_{\mathrm{ext}}^{2}
\end{array}\right\} \Rightarrow \Delta n^{(\mathrm{EK})}=3 A_{e} B_{\mathrm{ext}}^{2}=3.96 \times 10^{-24}\left(\frac{B_{\mathrm{ext}}}{1 \mathrm{~T}}\right)^{2} .
$$

The velocity of light in vacuum in the presence of an external field is less than $c$ and it depends on the polarization direction of the propagating wave: vacuum is predicted to behave as a uniaxial nonlinear crystal. The resulting birefringence predicted by $\mathcal{L}_{\mathrm{EK}}$ in the presence of an external magnetic field is extremely small [11-16].

Similarly, an electric field could be used to generate a birefringence in which case

$$
n_{\|}-n_{\perp}=\Delta n^{(\mathrm{EK})}=-3 A_{e}\left(\frac{E_{\mathrm{ext}}}{c}\right)^{2}
$$

but experimentally higher values of $B_{\mathrm{ext}}^{2}$ can be obtained with respect to $\left(E_{\mathrm{ext}} / c\right)^{2}$.

Generalizing to Post-Maxwellian nonlinear electrodynamics, given a Lagrangian density to second order in the invariants $\left(E^{2} / c^{2}-B^{2}\right)$ and $(\vec{E} / c \cdot \vec{B})$ of the form

$$
\mathcal{L}=\mathcal{L}_{\mathrm{Cl}}+\frac{\xi}{\mu_{0}}\left[\eta_{1}\left(\frac{E^{2}}{c^{2}}-B^{2}\right)^{2}+\eta_{2}\left(\frac{\vec{E}}{c} \cdot \vec{B}\right)^{2}\right]
$$

the vacuum magnetic birefringence resulting from Equations (8) and (9) is

$$
n_{\|}-n_{\perp}=\Delta n^{(\mathrm{PM})}=\xi\left(\eta_{2}-4 \eta_{1}\right) B_{\mathrm{ext}}^{2} .
$$


For example, in the Born-Infeld theory [20-22], originally introduced to limit the electrostatic self-energy of a point-like source, the Lagrangian is

$$
\mathcal{L}_{\mathrm{BI}}=\frac{b^{2}}{c^{2} \mu_{0}}\left[1-\sqrt{1-\frac{c^{2}}{b^{2}}\left(\frac{E^{2}}{c^{2}}-B^{2}\right)-\frac{c^{4}}{b^{4}}\left(\frac{\vec{E}}{c} \cdot \vec{B}\right)^{2}}\right]
$$

which expanded to second order in the invariants $\left(E^{2} / c^{2}-B^{2}\right)$ and $(\vec{E} / c \cdot \vec{B})$ results in

$$
\mathcal{L}_{\mathrm{BI}}=\mathcal{L}_{\mathrm{Cl}}+\frac{c^{2}}{8 b^{2} \mu_{0}}\left[\left(\frac{E^{2}}{c^{2}}-B^{2}\right)^{2}+4\left(\frac{\vec{E}}{c} \cdot \vec{B}\right)^{2}\right]+\ldots
$$

Here $b$ is the parameter of the theory and has the dimensions of an electric field. As can be seen $\eta_{2} / \eta_{1}=4$ resulting in a birefringence $\Delta n_{\mathrm{BI}}=0$ independently of $\xi=c^{2} / 8 b^{2}$. Note however that both $n_{\|, \perp}>1$ and light-by-light scattering is permitted.

\subsection{VMB beyond First Order QED}

The detection of VMB would be an extremely important verification of QED and its underlying bases. It would also demonstrate the validity of using low energy photons for particle physics in the $\mathrm{eV}-\mathrm{sub}-\mathrm{eV}$ domain. Several other effects, either predicted or hypothetical, could also be studied. Below are a few of these.

\subsubsection{Higher Order Corrections}

Radiative corrections to (10) due to QED have also been calculated [23] taking into account the electron-positron internal interaction depicted in Figure 2.

The resulting 2-loop contribution to the total Lagrangian is

$$
\mathcal{L}^{(\leq 2 \text { loop })}=\frac{\alpha}{36 \pi} \frac{A_{e}}{\mu_{0}}\left[160\left(\frac{E^{2}}{c^{2}}-B^{2}\right)^{2}+1315\left(\frac{\vec{E}}{c} \cdot \vec{B}\right)^{2}\right]
$$

resulting, from Equation (13), in a total birefringence

$$
\Delta n^{(\mathrm{EK})}+\Delta n^{(\leq 2 \text { loop })}=3 A_{e} B_{\text {ext }}^{2}\left(1+\frac{25 \alpha}{4 \pi}\right)=4.02 \times 10^{-24}\left(\frac{B_{\text {ext }}}{1 \mathrm{~T}}\right)^{2} .
$$

The 2-loop contribution consists of a relative increase with respect to the EulerHeisenberg-Kockel value of $\delta(\Delta n) / \Delta n=\frac{25 \alpha}{4 \pi}=1.45 \%$.
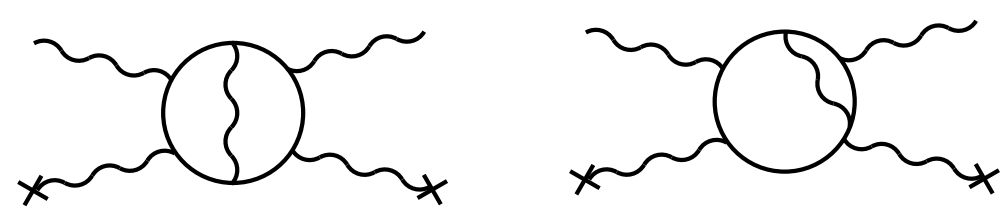

Figure 2. Example of two Feynman diagrams representing radiative corrections to vacuum magnetic birefringence due to the $e^{+} e^{-}$interaction.

\subsubsection{MilliCharged Particles (MCPs)}

Consider now vacuum fluctuations of hypothetical particles with charge $\pm \epsilon e$ and mass $m_{\epsilon}$ as discussed in [24,25]. Photons traversing a magnetic field may interact with such fluctuations resulting in a phase delay and, for photon energies $\hbar \omega>2 m_{\epsilon} c^{2}$, in a millicharged pair production. Therefore, a birefringence and a dichroism will result if such hypothetical particles exist. The cases of Dirac fermions (Df) and of scalar (sc) bosons here are considered separately. The indices of refraction for light polarized respectively parallel 
and perpendicular to the external magnetic field have two different mass regimes defined by the dimensionless parameter $\chi$ :

$$
\chi \equiv \frac{3}{2} \frac{\hbar \omega}{m_{\epsilon} c^{2}} \frac{\epsilon e B_{\mathrm{ext}} \hbar}{m_{\epsilon}^{2} c^{2}} .
$$

In the case of fermions, it can be shown that $[25,26]$

$$
\Delta n^{(\mathrm{Df})}=A_{\epsilon} B_{\text {ext }}^{2} \begin{cases}3 & \text { for } \chi \ll 1 \\ -\frac{9}{7} \frac{45}{2} \frac{\pi^{1 / 2} 2^{1 / 3}\left[\Gamma\left(\frac{2}{3}\right)\right]^{2}}{\Gamma\left(\frac{1}{6}\right)} \chi^{-4 / 3} & \text { for } \chi \gg 1\end{cases}
$$

where

$$
A_{\epsilon}=\frac{2}{45 \mu_{0}} \frac{\hbar^{3}}{m_{\epsilon}^{4} c^{5}} \epsilon^{4} \alpha^{2}
$$

in analogy to Equation (4). Please note that in the limit of large masses $(\chi \ll 1)$ expression (19) reduces to Equation (10) with the substitution of $\epsilon e$ with $e$ and $m_{\epsilon}$ with $m_{e}$. For small masses $(\chi \gg 1)$ the birefringence depends on the parameter $\chi^{-4 / 3}$ therefore resulting in a net dependence of $\Delta n^{(\mathrm{Df})}$ with $B_{\mathrm{ext}}^{2 / 3}$ rather than $B_{\mathrm{ext}}^{2}$ as in Equation (10). For dichroism one finds $[25,27]$

$$
\Delta \kappa^{(\mathrm{Df})}=\frac{1}{8 \pi} \frac{\epsilon^{3} e \alpha \lambda B_{\text {ext }}}{m_{\epsilon} c} \begin{cases}\sqrt{\frac{3}{32}} e^{-4 / \chi} & \text { for } \chi \ll 1 \\ \frac{2 \pi}{3 \Gamma\left(\frac{1}{6}\right) \Gamma\left(\frac{13}{6}\right)} \chi^{-1 / 3} & \text { for } \chi \gg 1 .\end{cases}
$$

Very similar results are found for the case of scalar millicharged particles [25]. Again, there are two mass regimes defined by the same parameter $\chi$ of expression (18). In this case, the magnetic birefringence is

$$
\Delta n^{(\mathrm{sc})}=A_{\epsilon} B_{\mathrm{ext}}^{2} \begin{cases}-\frac{6}{4} & \text { for } \chi \ll 1 \\ \frac{9}{14} \frac{45}{2} \frac{\pi^{1 / 2} 2^{1 / 3}\left[\Gamma\left(\frac{2}{3}\right)\right]^{2}}{\Gamma\left(\frac{1}{6}\right)} \chi^{-4 / 3} & \text { for } \chi \gg 1\end{cases}
$$

and the dichroism is

$$
\Delta \kappa^{(\mathrm{sc})}=\frac{1}{8 \pi} \frac{\epsilon^{3} e \alpha \lambda B_{\mathrm{ext}}}{m_{\epsilon} \mathcal{C}} \begin{cases}-\sqrt{\frac{3}{8}} e^{-4 / \chi} & \text { for } \chi \ll 1 \\ -\frac{\pi}{3 \Gamma\left(\frac{1}{6}\right) \Gamma\left(\frac{13}{6}\right)} \chi^{-1 / 3} & \text { for } \chi \gg 1 .\end{cases}
$$

As can be seen, there is a sign difference with respect to the case of Dirac fermions, both for the induced birefringence and the induced dichroism.

Vacuum magnetic birefringence and vacuum magnetic dichroism (VMD) limits can therefore constrain the existence of such millicharged particles.

\subsubsection{Axion-Like Particles (ALPs)}

The propagation of light in an external electromagnetic field could also depend on the existence of hypothetical light neutral particles coupling to two photons. The involved processes are shown in Figure 3: the production diagram implies an absorption of light quanta, whereas a phase delay is produced by the recombination process. The search for such particles with masses below $\sim 1 \mathrm{eV}$ has recently gained much attention as these are considered to be good candidates for particle dark matter. 

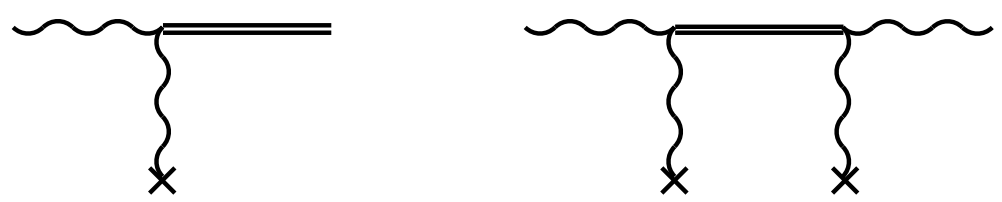

Figure 3. Axion-like particle interaction with two photons through the Primakoff effect [28]. (Left) first order production. (Right) second order recombination.

In 1977, to solve the strong CP problem, i.e., the non observation of CP violation within the strong interactions, Peccei and Quinn (PQ) $[29,30]$ introduced a new global symmetry which, when broken at high energy, gives rise to a pseudo-Nambu-Goldstone boson called the axion [31-33]. The value of its mass is not predicted while the couplings to the standard model particles are defined by the model implementing the PQ symmetry: couplings are generally very weak and proportional to the mass of the axion.

To broaden the idea of the axion, a more general class of Axion-Like Particles (ALPs) has also been introduced. In this case, the mass and coupling constant are independent of each other. Axions and ALPs have been searched for in dedicated experiments since their proposal [34], but to date these remain undetected and in the case of ALPs only a fraction of the available mass-coupling constant space has been probed: present and future experiments cover the mass range starting from values as low as $10^{-22} \mathrm{eV}$ up to several gigaelectronvolt with a more favorable window in the mass range between $10 \mu \mathrm{eV}$ and $1 \mathrm{meV}$.

Most experimental searches rely on the axion-photon coupling mediated by a twophoton vertex shown in Figure 3. Other searches are based on the axion-electron interaction, present through an axion-spin interaction, but only in some models such as the Dine-Fishler-Sredincki-Zhitnitsky (DFSZ) [35,36] one. A review of experimental efforts to search for ALPs and the axion can be found in $[37,38]$.

The Lagrangian densities describing the interaction of ALPs with two photons, for convenience expressed in natural Heaviside-Lorentz units ${ }^{1}$, can be written as

$$
\mathcal{L}_{a}=g_{a} a \vec{E} \cdot \vec{B} \quad \text { and } \quad \mathcal{L}_{s}=g_{s} s\left(E^{2}-B^{2}\right)
$$

where $g_{a}$ and $g_{s}$ are the coupling constants to two photons of the pseudoscalar field $a$ or scalar field $s$, respectively. Therefore, for the pseudoscalar case, in the presence of an external uniform magnetic field $\vec{B}_{\text {ext }}$ only the component of the electric field of light $\vec{E}_{\gamma}$ parallel to $\vec{B}_{\text {ext }}$ will interact with the pseudoscalar field. For the scalar case, the opposite is true: an interaction is only possible for the component of $\vec{E}_{\gamma}$ normal to $\vec{B}_{\text {ext }}$.

For photon energies above the mass of such particle candidates a real production can follow leading to a dichroism $\Delta \kappa$. Even if the photon energy is smaller than the particle mass virtual production can follow leading to a reduction of the speed of light of one polarization component with respect to the other resulting in a birefringence $\Delta n$.

Therefore in the pseudoscalar case, for which $\vec{E}_{\gamma} \cdot \vec{B}_{\text {ext }} \neq 0$, one has $n_{\|}^{a}>1, \kappa_{\|}^{a}>0$, $n_{\perp}^{a}=1$ and $\kappa_{\perp}^{a}=0$ whereas in the scalar case for which $\vec{B}_{\gamma} \cdot \vec{B}_{\text {ext }} \neq 0$ one finds $n_{\perp}^{s}>1$, $\kappa_{\perp}^{s}>0, n_{\|}^{s}=1$ and $\kappa_{\|}^{s}=0$. It can be shown that the dichroism $\Delta \kappa^{(\mathrm{ALPs})}$ and the birefringence $\Delta n^{(\mathrm{ALPs})}$ due to the existence of such bosons can be expressed as [39-42]:

$$
\begin{aligned}
& \left|\Delta \kappa^{(\mathrm{ALPs})}\right|=\kappa_{\|}^{a}=\kappa_{\perp}^{s}=\frac{2}{\omega L_{B}}\left(\frac{g_{a, s} B_{\mathrm{ext}} L_{B}}{4}\right)^{2}\left(\frac{\sin x}{x}\right)^{2} \\
& \left|\Delta n^{(\mathrm{ALPs})}\right|=n_{\|}^{a}-1=n_{\perp}^{s}-1=\frac{1}{2}\left(\frac{g_{a, s} B_{\mathrm{ext}}}{2 m_{a, s}}\right)^{2}\left(1-\frac{\sin 2 x}{2 x}\right)
\end{aligned}
$$


where in vacuum the adimensional parameter $x=\frac{L_{B} m_{a, s}^{2}}{4 \omega}, \omega$ is the photon energy in $\mathrm{eV}$, $L_{B}$ is the external magnetic field length in units $\mathrm{eV}^{-1}$ and the field strength is $B_{\mathrm{ext}} \mathrm{in} \mathrm{eV}^{2}$. In the approximation $x \ll 1$ (small masses) expressions (24) and (25) become

$$
\begin{aligned}
& |\Delta \kappa|=\kappa_{\|}^{a}=\kappa_{\perp}^{s}=\frac{2}{\omega L_{B}}\left(\frac{g_{a, s} B_{\mathrm{ext}} L_{B}}{4}\right)^{2} \\
& |\Delta n|=n_{\|}^{a}-1=n_{\perp}^{s}-1=\frac{1}{3}\left(\frac{g_{a, s} B_{\mathrm{ext}} m_{a, s} L_{B}}{4 \omega}\right)^{2}
\end{aligned}
$$

where it is interesting to note that $\Delta \kappa$ in this case is independent of $m_{a, s}$. For $x \gg 1$

$$
\begin{aligned}
& |\Delta \kappa|=\kappa_{\|}^{a}=\kappa_{\perp}^{s}<2 \omega\left(\frac{g_{a, s} B_{\mathrm{ext}}}{m_{a, s}^{2}}\right)^{2} \\
& |\Delta n|=n_{\|}^{a}-1=n_{\perp}^{s}-1=\frac{1}{2}\left(\frac{g_{a, s} B_{\mathrm{ext}}}{m_{a, s}}\right)^{2} .
\end{aligned}
$$

Please note that the birefringence induced by pseudoscalars and scalars are opposite in sign: $n_{\|}^{a}>n_{\perp}^{a}=1$ whereas $n_{\|}^{s}=1<n_{\perp}^{s}$.

Optical polarimetry to detect $\Delta \kappa^{(\mathrm{ALPs})}$ and $/$ or $\Delta n^{(\mathrm{ALPs})}$ was experimentally pioneered by the BFRT collaboration [40] and subsequently continued by the long lasting PVLAS collaboration with an apparatus first installed at INFN National Laboratories in Legnaro (LNL), Italy [43-45] and subsequently at the INFN section of Ferrara, Italy. The detection of an ALPs-induced birefringence and dichroism would allow the determination of the mass and coupling constant of the ALPs to two photons.

Other laboratory optical experiments are the so-called "light shining through a wall" (LSW) experiments, where a regeneration scheme is employed [46-51].

\section{Experimental Method}

As shown in the previous section, the VMB value in expression (10) to be measured is extremely small. This section will discuss in detail the experimental method to measure such a tiny quantity. The principle of the method described below was proposed for the first time in 1979 by E. Iacopini and E. Zavattini [52].

In general, to measure the linear birefringence induced by an external field a linearly polarized beam of light is propagated across the birefringent medium. For a non-zero angle $\vartheta$ between the initial polarization direction and the direction of the external field, which defines the optical axis of the birefringent medium, the light becomes elliptically polarized, namely the tip of the electric field describes an ellipse. This process is schematized in Figure 4. The ratio of the minor to the major axis of the ellipse is the ellipticity $\psi= \pm a / b$ where the sign distinguishes between the two rotation directions of the electric field vector. We are interested in the case in which the phase difference $\Delta \phi$ acquired by the components of the electric field in the natural reference system of the birefringent medium is small:

$$
\Delta \phi=\frac{2 \pi}{\lambda} \int \Delta n d z \ll 1 .
$$

In the formalism of the Jones' matrices the evolution of the electric field of the light can be written as

$$
\mathbf{E}_{\text {out }}=\mathbf{R}(-\vartheta) \cdot \mathbf{M}(\Delta \phi) \cdot \mathbf{R}(\vartheta) \cdot\left(\begin{array}{c}
E_{\text {in }} \\
0
\end{array}\right) \approx E_{\text {in }}\left(\begin{array}{c}
1+i \frac{\Delta \phi}{2} \cos 2 \vartheta \\
i \frac{\Delta \phi}{2} \sin 2 \vartheta
\end{array}\right)
$$

where

$$
\mathbf{R}(\vartheta)=\left(\begin{array}{cc}
\cos \vartheta & \sin \vartheta \\
-\sin \vartheta & \cos \vartheta
\end{array}\right) \quad \text { and } \quad \mathbf{M}(\Delta \phi)=\left(\begin{array}{cc}
e^{i \Delta \phi / 2} & 0 \\
0 & e^{-i \Delta \phi / 2}
\end{array}\right)
$$


are the rotation matrix and the birefringent medium, respectively. For $|\Delta \phi| \ll 1$ Equation (31) is the normal equation of an ellipse and the ratio of the minor to major axes of the ellipse is the ellipticity $\psi(\vartheta)= \pm a / b=(\Delta \phi / 2) \sin 2 \vartheta$ with maximum ellipticity $|\psi|=|\Delta \phi / 2|$ for $\vartheta= \pm \pi / 4(\bmod \pi)$ :

$$
\mathbf{E}_{\mathrm{out}}=E_{\mathrm{in}}\left(\begin{array}{c}
1+i \psi \cos 2 \vartheta \\
i \psi \sin 2 \vartheta
\end{array}\right) .
$$

It can be shown that the polarization does not rotate to first order in $\psi$. Note also that $E_{Y, \text { out }}$ has a phase delay of $\pi / 2$ with respect to $E_{X, \text { out }}$.

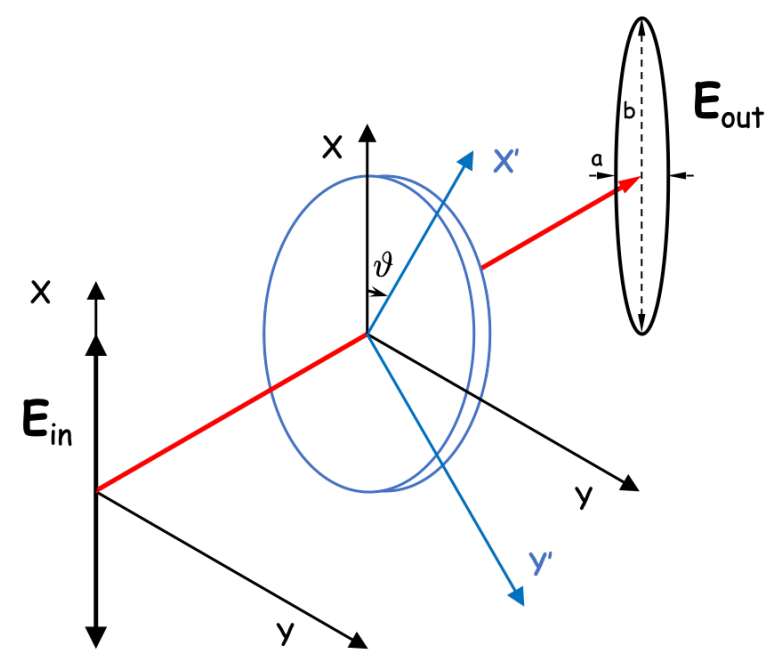

Figure 4. Polarization evolution of a light beam traversing a birefringent medium. The $X Y Z$ reference frame is defined by the initial polarization vector $\mathbf{E}_{\text {in }}$ and the wave-vector $\mathbf{k}$. The rotated axes $X^{\prime} Y^{\prime} Z$ coincide with the birefringence axes of the optical medium.

An identical treatment can be followed in the case of a linear dichroism $\Delta \kappa$. We are interested in the case in which the difference in relative amplitude reduction $\Delta \zeta$ accumulated by a linearly polarized beam passing through a dichroic medium is small:

$$
\Delta \zeta=\frac{2 \pi}{\lambda} \int \Delta \kappa d z \ll 1
$$

In this case, the electric field exiting a dichroic medium will be

$$
\mathbf{E}_{\text {out }} \approx E_{\text {in }}\left(\begin{array}{c}
1+\frac{\Delta \zeta}{2} \cos 2 \vartheta \\
\frac{\Delta \zeta}{2} \sin 2 \vartheta
\end{array}\right) \approx E_{\text {in }}\left(\begin{array}{c}
1 \\
\varphi \sin 2 \vartheta
\end{array}\right)
$$

where it is apparent that, to first order, $\mathbf{E}_{\text {out }}$ is rotated by an angle $\varphi(\vartheta)=-(\Delta \zeta / 2) \sin 2 \vartheta$ with respect to $\mathbf{E}_{\text {in }}$ and that $E_{Y \text {,out }}$ is in phase with respect to $E_{X \text {,out }}$.

From the above discussion it is also clear that ellipticities and rotations induced by different media will add up algebraically as complex numbers so long as $\psi, \varphi \ll 1$ :

$$
\xi=\varphi+i \psi
$$

Furthermore, it is evident that $\xi$ can be increased by lengthening the optical path within the optical medium.

The scheme of a high sensitivity polarimeter designed to measure very small ellipticities and rotations is shown in Figure 5. An input polarizer defines the polarization of the light entering the magnetic field region; this is the $\|$ direction. To increase the optical path length within the magnetic field of length $L_{B}$ this field region is enclosed between the two mirrors of a Fabry-Perot interferometer. The Fabry-Perot cavity is a multiple-beam 
interference device [53]. The electric field of the transmitted beam depends on the optical path length between the two mirrors in a way described by the Airy curve:

$$
E_{T}(\delta)=E_{\mathrm{in}} \frac{T e^{i \delta / 2}}{1-R e^{i \delta}}
$$

where $\delta=4 \pi n L / \lambda$ is the phase accumulated by the light for a round-trip between the mirrors of reflectance $R \lesssim 1$ (assumed to be identical) and $T=1-R$ (no losses). The resonance is maximum for $\delta=2 m \pi$. For small deviations of $\delta$ from $2 m \pi, E_{T}(\delta)$ will acquire an amplified phase shift $\Phi=\delta \frac{1+R}{1-R} \approx \delta 2 \mathcal{F} / \pi$ where $\mathcal{F}$ defines the finesse of the cavity as $\mathcal{F}=\pi /(1-R)$. The equivalent number of passes through the magnetic field results in $N=2 \mathcal{F} / \pi$ and can be as high as $N \approx 5 \times 10^{5}$. The cavity is kept on the top of the resonance by means of a feedback system [54-56] which dynamically modifies the laser frequency to match the resonance condition for $\mathbf{E}_{\mathrm{in}}$. For this purpose, the necessary light reflected from the cavity is sampled immediately out of the input polarizer. Due to the magnetic birefringence $\Delta n$ and/or dichroism $\Delta \kappa$ the field component $E_{Y^{\prime}}$ will be phase shifted and/or attenuated with respect to $E_{X^{\prime}}$. The Fabry-Perot will therefore multiply $\xi=\varphi+i \psi$ by a factor $N$ resulting in a total effect

$$
\Xi=N \xi=N(\varphi+i \psi)=\Phi+i \Psi .
$$

One has then

$$
\mathbf{E}_{\text {out }} \approx \pm E_{\text {in }} \frac{T}{1-R}\left[\begin{array}{c}
1 \\
(\Phi+i \Psi) \sin 2 \vartheta
\end{array}\right]
$$

where Equation (35) has been used.

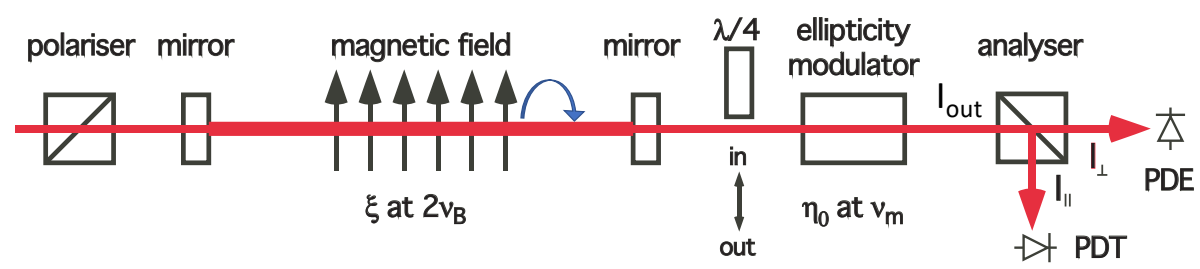

Figure 5. Schematic drawing of the PVLAS polarimeter. A rotating magnetic field between the cavity mirrors generates a time-dependent ellipticity. The laser frequency is phase-locked to the cavity resonance; the locking system (not shown in figure) uses the power reflected from the cavity exiting the input polarizer along the input path.

To further increase the sensitivity for detecting VMB and/or VMD the magnetic field is rotated at a frequency $v_{B} \sim 1-10 \mathrm{~Hz}$ resulting in $\vartheta(t)=2 \pi v_{B} t+\vartheta_{B}$. Following the magnetic field region is an ellipticity modulator, generating a known time-dependent carrier ellipticity $\eta(t)=\eta_{0} \cos \left(2 \pi v_{m} t+\theta_{m}\right)$, and the crossed analyzer set to maximum extinction. The power detected on the photodiode PDE will therefore be

$$
I_{\perp}^{(\mathrm{ell})}=I_{\mathrm{out}}|i \eta(t)+(\Phi+i \Psi) \sin 2 \vartheta(t)|^{2} \approx I_{\|}\left[\eta(t)^{2}+2 \eta(t) \Psi \sin 2 \vartheta(t)+\ldots\right]
$$

where we have approximated $I_{\text {out }} \approx I_{\|}$. In Equation (37) the dots indicate higher order terms in $\Phi$ and $\Psi$. In general, the field intensity may be varied with a fixed $\vartheta$ but since both the LNL and Ferrara versions of the PVLAS experiment have always rotated the magnetic field, expression (37) makes it clear that the sought for effect will appear at twice the rotation frequency of the magnetic field.

During the years of the PVLAS collaboration two different ellipticity modulators were used: a commercial (Hinds Instruments) resonant photo-elastic modulator (PEM) with $v_{m} \approx 20 \mathrm{kHz}$ and $50 \mathrm{kHz}$ depending on its size and a non-resonant stress-optic modulator (SOM) [57] with $v_{m}=400 \div 600 \mathrm{~Hz}$. 
It is important to note that on the right-hand side of expression (37) the ellipticity $\eta(t)$ will not beat with the rotation $\Phi \sin 2 \vartheta(t)$ given that one is imaginary, and the other is real. In Figure 5 the presence of the insertable quarter-wave plate (QWP) is precisely there for rotation measurements: the QWP transforms a rotation $\Phi \sin 2 \vartheta(t)$ into an ellipticity $\pm i \Phi \sin 2 \vartheta(t)$, the sign depending on the orientation of the QWP.

Experimentally two other considerations must be made during ellipticity measurements: the polarizer and analyzer have an intrinsic non-zero extinction ratio $I_{\perp} / I_{\|}=\sigma^{2}$ and an ellipticity noise $\gamma(t)=\gamma_{0}(t)+N \gamma_{\text {cavity }}(t)$. Indeed $\gamma(t)$ will have contributions $\gamma_{0}(t)$ from outside the cavity and $N \gamma_{\text {cavity }}(t)$ from the cavity mirrors which will therefore depend on $N$, as will be discussed in Section 2.3. Considering the case of no magnetically induced dichroism, therefore $\Phi=0$, Equation (37) is modified to

$$
I_{\perp}^{(\mathrm{ell})}=I_{\|}\left[\sigma^{2}+\eta(t)^{2}+2 \eta(t) \Psi \sin 2 \vartheta(t)+2 \eta(t) \gamma(t)+\ldots\right] .
$$

Vice versa in the presence of a dichroism and with the QWP inserted but in the absence of birefringence Equation (38) will become

$$
I_{\perp}^{(\mathrm{rot})}=I_{\|}\left[\sigma^{2}+\eta(t)^{2} \pm 2 \eta(t) \Phi \sin 2 \vartheta(t) \pm 2 \eta(t) \Gamma(t)+\ldots\right]
$$

where again one must also include the rotation noise $\Gamma(t)$ and the signs depend on the orientation of the QWP.

The power $I_{\perp}$ is collected on a low noise InGaAs photo detector with an efficiency of $q=0.7 \mathrm{~A} / \mathrm{W}$ at $\lambda=1064 \mathrm{~nm}$ and a typical gain of $G=10^{6} \mathrm{~V} / \mathrm{A}$.

The sought for values of the quantities $\Psi$ or $\Phi$ are extracted from Equations (38) or (39) by demodulating the extinguished power $I_{\perp}$ at $v_{m}$ and $2 v_{m}$. The DC component of the demodulated signal at $2 v_{m}$ derives from the term $I_{\|} \eta(t)^{2}$ and results in $I_{2 v_{m}}=I_{\|} \eta_{0}^{2} / 2$ from which $\eta_{0}$ is determined whereas the demodulated output signal at $v_{m}$ will contain a component at $2 v_{B}$, sum of the two sidebands $I_{ \pm}$at $v_{m} \pm 2 v_{B}$ deriving from the product $2 \eta(t) \Psi \sin 2 \vartheta(t): I_{2 v_{B}}=I_{+}+I_{-}=2 I_{\|} \eta_{0} \Psi$ at $2 v_{B}$ (or $I_{2 v_{B}}=2 I_{\|} \eta_{0} \Phi$ for rotation measurements). The resulting ellipticity and rotation can therefore be extracted as functions of the measured quantities $I_{2 v_{m}}$ and $I_{2 v_{B}}$ :

$$
\Psi, \Phi=\frac{I_{2 v_{B}}}{2 \sqrt{2 I_{\|} I_{2 v_{m}}}}=\frac{I_{2 v_{B}}}{2 \eta_{0} I_{\|}}=\frac{I_{2 v_{B}}}{I_{2 v_{m}}} \frac{\eta_{0}}{4} .
$$

The ellipticity and the rotation come with a well-defined phase $2 \vartheta_{B}$ such that $\Psi$ or $\Phi$ are maximum for $\vartheta= \pm \pi / 4(\bmod \pi)$.

Following the definition of ellipticity

$$
\Psi=N \frac{\pi}{\lambda} \int \Delta n d z \sin 2 \vartheta
$$

an estimate of the expected VMB signal, considering $N=5 \times 10^{5}, \int_{L_{B}} B_{\mathrm{ext}}^{2} d z \approx 10 \mathrm{~T}^{2} \mathrm{~m}$, $\lambda=1064 \mathrm{~nm}$ and $\vartheta=\pi / 4$, is

$$
\Psi^{(\mathrm{EK})}=N \frac{\pi}{\lambda} \int_{L_{B}} 3 A_{e} B_{\mathrm{ext}}^{2} d z=6 \times 10^{-11} .
$$

These were the experimental parameters of the final version of the PVLAS experiment.

\subsection{Systematic Effects}

A well-known systematic of this polarimeter (see for example Refs. [1,58,59]) is generated by the sum of two intrinsic effects. The first is the fact that the dielectric mirrors employed to make the Fabry-Perot cavity exhibit a slight birefringence on reflection. The two mirrors are equivalent to a single wave plate, whose phase difference $\delta_{\mathrm{EQ}}$ is amplified by the many passages in the cavity. In principle, this is a static effect not interfering 
with heterodyne polarimetry. Secondly, the azimuthal angle of the two mirrors is normally adjusted so as the axis of the equivalent wave plate is aligned to the input polarization. In this condition, however, the two orthogonal polarizations inside the Fabry-Perot are no longer equivalent. Although the $\|$ one is locked on the top of the resonance curve by the laser phase-locking system, the other, which has a different round-trip phase $\delta_{\mathrm{EQ}} \neq 0$, is out of resonance. Due to $\delta_{\mathrm{EQ}}$, instead of having Equation (36) one finds

$$
\mathbf{E}_{\text {out }} \approx \pm E_{\text {in }} \frac{T}{1-R}\left(\begin{array}{c}
1 \\
(\Phi+i \Psi)\left(1+i \Delta_{\mathrm{EQ}}\right) \sin 2 \vartheta
\end{array}\right)
$$

where $\Delta_{\mathrm{EQ}}=N \delta_{\mathrm{EQ}}$. According to this equation, there is a cross talk between the ellipticity and rotation measurements: even if $\Phi=0$ there is a real component in $E_{Y, o u t}$ describing a rotation. Instead of Equations (38) and (39) one finds

$$
I_{\perp}^{(\mathrm{ell})}(\phi)=I_{\|}\left[\sigma^{2}+\eta(t)^{2}+\eta(t) k\left(\delta_{\mathrm{EQ}}\right)\left(2 \Psi+\Phi \Delta_{\mathrm{EQ}}\right) \sin 2 \vartheta(t)+\ldots\right],
$$

and

$$
I_{\perp}^{(\mathrm{rot})}(\phi)=I_{\|}\left[\sigma^{2}+\eta(t)^{2} \pm \eta(t) k\left(\delta_{\mathrm{EQ}}\right)\left(2 \Phi-\Psi \Delta_{\mathrm{EQ}}\right) \sin 2 \vartheta(t)+\ldots\right],
$$

where the factor

$$
k\left(\delta_{\mathrm{EQ}}\right)=\frac{1}{1+N^{2} \sin ^{2}\left(\delta_{\mathrm{EQ}} / 2\right)} \leq 1
$$

is due to the fact that the $\perp$ polarization is out of resonance. To disentangle the ellipticity and the rotation, both quantities must be measured in the same experimental conditions. The cavity parameter $\delta_{\mathrm{EQ}}$ must be determined in a calibration with known signals.

Generally, there is no dichroism induced by a magnetic field and $\Phi=0$. Therefore, during calibration measurements with gases described in Section 2.4, by measuring both the ellipticity signal $\Psi$ and the 'spurious' rotation $\Phi^{\text {(spurious) }}=\mp \Psi \Delta_{\mathrm{EQ}} / 2$ one can determine directly the value of $\Delta_{\mathrm{EQ}}$ :

$$
R=\left|\frac{\Phi^{\text {(spurious) }}}{\Psi}\right|=\frac{\left|\Delta_{\mathrm{EQ}}\right|}{2} .
$$

\subsection{Noise Considerations}

Consider the output signal from the lock-in which demodulates at $v_{m}$. If the rms intensity noise $S_{I_{-}}$at the frequency $v_{m}-2 v_{B}$ is uncorrelated with the rms intensity noise $S_{I_{+}}$at $v_{m}+2 v_{B}$ and $S_{I_{+}}=S_{I_{-}} \equiv S_{I_{ \pm}}$, the demodulated rms intensity noise will be $S_{I_{2 v_{B}}}=\sqrt{S_{I_{+}}^{2}+S_{I_{-}}^{2}}=\sqrt{2} S_{I_{ \pm}}$due to the folding of the spectrum around $v_{m}$. Using Equation (40) the expected peak ellipticity sensitivity $S_{\Psi_{2 v_{B}}}$ of the polarimeter is

$$
S_{\Psi_{2 v_{B}}}=\frac{S_{I_{2 v_{B}}}}{I_{\|} \eta_{0}}
$$

Several intrinsic effects contribute to $S_{I_{2 v_{B}}}$ all of which can be expressed as a noise in the light power $I_{\perp}$. First consider the intrinsic rms shot-noise spectral density due to the direct current $i_{\mathrm{dc}}$ in the detector

$$
S_{i}^{(\text {shot })}=\sqrt{2 e i_{\mathrm{DC}}}=\sqrt{2 e q I_{\perp, D C}}
$$

where $q$ is the efficiency of the photo detector in amperes/watts and $S_{i}^{\text {(shot) }}$ is measured in

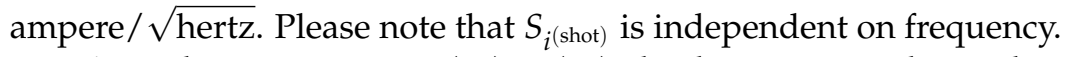

According to Equations (38) or (39), the direct current due to the modulator inside the photodiode is $i_{\mathrm{DC}}=q I_{\|} \eta_{0}^{2} / 2$. Taking also into account the contribution of the extinction ratio of the polarizers, which can be as low as $\sigma^{2} \lesssim 10^{-7}$, and of the square of the static 
component of the ellipticity noise $\gamma(t), \gamma_{D C}$, this effect introduces an additional term in the detected DC power which is written as $I_{\|}\left(\sigma^{2}+\gamma_{\mathrm{DC}}^{2}\right)$. This leads to an expression for the shot-noise spectral densities in the light power $S_{I}^{(\text {shot })}$ and in the ellipticity $S_{\Psi}^{(\text {shot })}$

$$
\begin{aligned}
& S_{I}^{(\text {shot })}=\frac{S_{i}^{(\text {shot })}}{q}=\sqrt{\frac{2 e I_{\|}}{q}\left(\sigma^{2}+\gamma_{\mathrm{DC}}^{2}+\frac{\eta_{0}^{2}}{2}\right)} \text { and } \\
& S_{\Psi}^{\text {(shot })}=\sqrt{\frac{2 e}{q I_{\|}}\left(\frac{\sigma^{2}+\gamma_{\mathrm{DC}}^{2}+\eta_{0}^{2} / 2}{\eta_{0}^{2}}\right) .}
\end{aligned}
$$

Other effects contributing to the power and ellipticity noise spectral densities are the Johnson noise of the transimpedance $G$ of the photo detector amplifier

$$
S_{I}^{(\mathrm{J})}=\sqrt{\frac{4 k_{B} T}{q^{2} G}}, \quad \text { giving } \quad S_{\Psi}^{(\mathrm{J})}=\sqrt{\frac{4 k_{B} T}{G}} \frac{1}{q I_{\|} \eta_{0}},
$$

the photodiode dark-current noise

$$
S_{I}^{(\text {dark })}=\frac{S_{i}^{(\text {dark })}}{q}, \quad \text { with } \quad S_{\Psi}^{(\text {dark })}=\frac{S_{i}^{(\text {dark })}}{q I_{\|} \eta_{0}}
$$

and the frequency dependent relative intensity noise $N_{v}^{(\mathrm{RIN})}$ of the light emerging from the cavity

$$
S_{I_{v}}^{(\mathrm{RIN})}=I_{\|} N_{v}^{(\mathrm{RIN})}
$$

giving

$$
S_{\Psi_{2 v_{B}}}^{(\mathrm{RIN})}=N_{v_{m}}^{(\mathrm{RIN})} \frac{\sqrt{\left(\sigma^{2}+\gamma_{\mathrm{DC}}^{2}+\eta_{0}^{2} / 2\right)^{2}+\left(\eta_{0}^{2} / 2\right)^{2}}}{\eta_{0}} .
$$

In the last equation we consider that the contribution of $I_{\mathrm{DC}}$ and $I_{2 v_{B}}$ in the Fourier spectrum add incoherently to the intensity noise at $v_{m} \pm 2 v_{B}$ with $v_{B} \ll v_{m}$.

Figure 6 shows all the intrinsic contributions as functions of $\eta_{0}$ in typical PVLAS-FE operating conditions, with $q=0.7 \mathrm{~A} / \mathrm{W}, I_{\|}=8 \mathrm{~mW}, \sigma^{2}=2 \times 10^{-7}, G=10^{6} \mathrm{~V} / \mathrm{A}$, $S_{i}^{\text {(dark) }}=25 \mathrm{fA}_{\mathrm{rms}} / \sqrt{\mathrm{Hz}}$, and $N_{v_{m}}^{(\mathrm{RIN})} \approx 3 \times 10^{-7} / \sqrt{\mathrm{Hz}} @ 50 \mathrm{kHz}$ (resonance frequency of the PEM). The figure shows that the expected total ellipticity noise

$$
S_{\Psi}^{(\text {tot })}=\sqrt{S_{\Psi}^{(\text {shot })^{2}}+S_{\Psi}^{(\mathrm{J})^{2}}+S_{\Psi}^{(\text {dark })^{2}}+S_{\Psi}^{(\mathrm{RIN})^{2}}}
$$

has a minimum for a modulation amplitude $\eta_{0} \approx 10^{-2}$ close to shot-noise. The above noise considerations are also valid in the case of rotation measurements.

It is important to note here that the signal to noise ratio sensitivity of the polarimeter will improve increasing $N$ only if $S_{\Psi}\left(\right.$ and $\left.S_{\Phi}\right)$ do not depend on the equivalent number of passes $N$ of the cavity. The three noise sources presented above, namely shot-noise, Johnson noise and dark-current noise, satisfy this condition depending in no way on the presence of the cavity. If, however, an ellipticity noise such as $\gamma_{\text {cavity }}(t)$ [see Equation (38) and nearby text] originating from inside the cavity is present and if $\pi N \gamma_{\text {cavity }}(t) / \lambda>S_{\Psi}^{(\text {tot })}$ both the magnetically induced signal and the noise would increase proportionally to $N$ thus the signal to noise ratio sensitivity will not improve. The same argument is true in the presence of a rotation noise $\Gamma_{\text {cavity }}(t)$ [see Equation (39)]. 


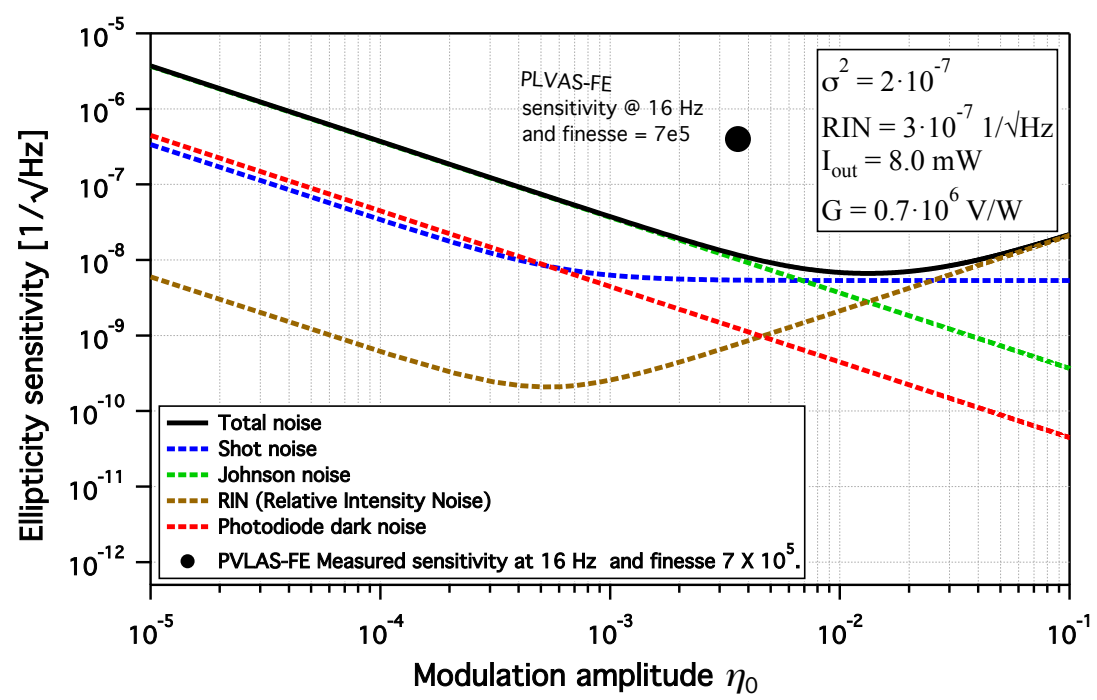

Figure 6. Intrinsic peak noise components of the polarimeter as a function of the ellipticity modulation amplitude $\eta_{0}$. Superimposed is the PVLAS-FE peak ellipticity noise at $16 \mathrm{~Hz}$ well above the expected sensitivity due to the Fabry-Perot mirrors (see Section 2.3).

Finally, the presence of the DC component of $\gamma(t)$ in Equation (38), $\gamma_{D C}$, may also contribute to an ellipticity noise $S_{\Psi}$ at the signal frequency $2 v_{B}$ in the presence of a relative intensity noise $N_{v}^{(\mathrm{RIN})}$ of $I_{\|}$at $2 v_{B}$. From Equation (38) the condition for which this effect will not deteriorate $S_{\Psi}$ is that the product $N_{v}^{(\mathrm{RIN})} \gamma_{\mathrm{DC}}$ satisfies

$$
N_{v}^{(\mathrm{RIN})} \gamma_{\mathrm{DC}} \ll S_{\Psi} .
$$

In PVLAS the typical relative intensity noise of $I_{\|}$in the frequency range $0.5 \div 20 \mathrm{~Hz}$ was $N_{v}^{(\mathrm{RIN})}=S_{I_{\|}} / I_{\|} \lesssim 10^{-4} / \sqrt{\mathrm{Hz}}$. By keeping $\gamma_{\mathrm{DC}} \lesssim 10^{-4}$ the contribution to $S_{\Psi}$ will be $N_{v}^{(\mathrm{RIN})} \gamma_{\mathrm{DC}} \lesssim 10^{-8}$. This was done with a very low-frequency feedback (cutoff frequency much lower than $2 v_{B}$ ) using the ellipticity signal at the output of the lock-in amplifier demodulating at $v_{m}$. In the PVLAS setup which used the non-resonant SOM the actuator was the SOM itself which could generate static ellipticities whereas in the PVLAS setup which used the PEM the feedback actuation was done with the input polarizer coupled to the cavity intrinsic birefringence as will be discussed in more detail below.

\subsection{Expected Detectability of $V M B$}

In principle, with the magnetic field and optical parameters leading to $\Psi^{(\mathrm{EK})}=6 \times 10^{-11}$ in Equation (42) and the optimal sensitivity in Figure 6 of $S_{\Psi}^{\text {(tot) }} \approx 8 \times 10^{-9} / \sqrt{\mathrm{Hz}}$, the VMB signal should have been detected in a time $T=\left(S_{\Psi}^{(\text {tot })} / \Psi^{(\mathrm{ED})}\right)^{2} \approx 5 \mathrm{~h}$ with a SNR $=1$.

Unfortunately shot-noise limited measurements with finesse values $\mathcal{F} \gtrsim 50,000$ and signal frequencies $v \lesssim 500 \mathrm{~Hz}$ have never been obtained in any polarimeter due to the presence of $N \gamma_{\text {cavity }}(t)$ originating from the cavity mirrors [1,60]. In Figure 7 one can see the sensitivity in optical path difference $S_{\Delta \mathcal{D}}$ of the different experimental efforts to measure VMB defined as

$$
S_{\Delta \mathcal{D}}=S_{\Psi} \frac{\lambda}{N \pi} .
$$

Considering the optical path difference sensitivity allows a direct comparison of the optical performance of the different experiments being $S_{\Delta \mathcal{D}}$ independent of $\lambda$ and $N$. The VMB induced optical path difference is $\Delta \mathcal{D}=3 A_{e} \int_{L_{B}} B_{\text {ext }}^{2} d L$. In the same graph, the shot-noise expected sensitivity for each experiment is also shown. A clear correlation described approximately by a power law can be seen between $S_{\Delta \mathcal{D}}$ and the signal frequency. The two almost equivalent points from BFRT [40] were measured with two 
different cavities, one with 34 passes and the other 578 passes whereas the two PVLAS-LNL superimposed points [45] are for $\lambda=1064 \mathrm{~nm}$ and $\mathcal{F}^{(1064)}=70,000$ and for $\lambda=532 \mathrm{~nm}$ and $\mathcal{F}^{(532)}=35,000$.

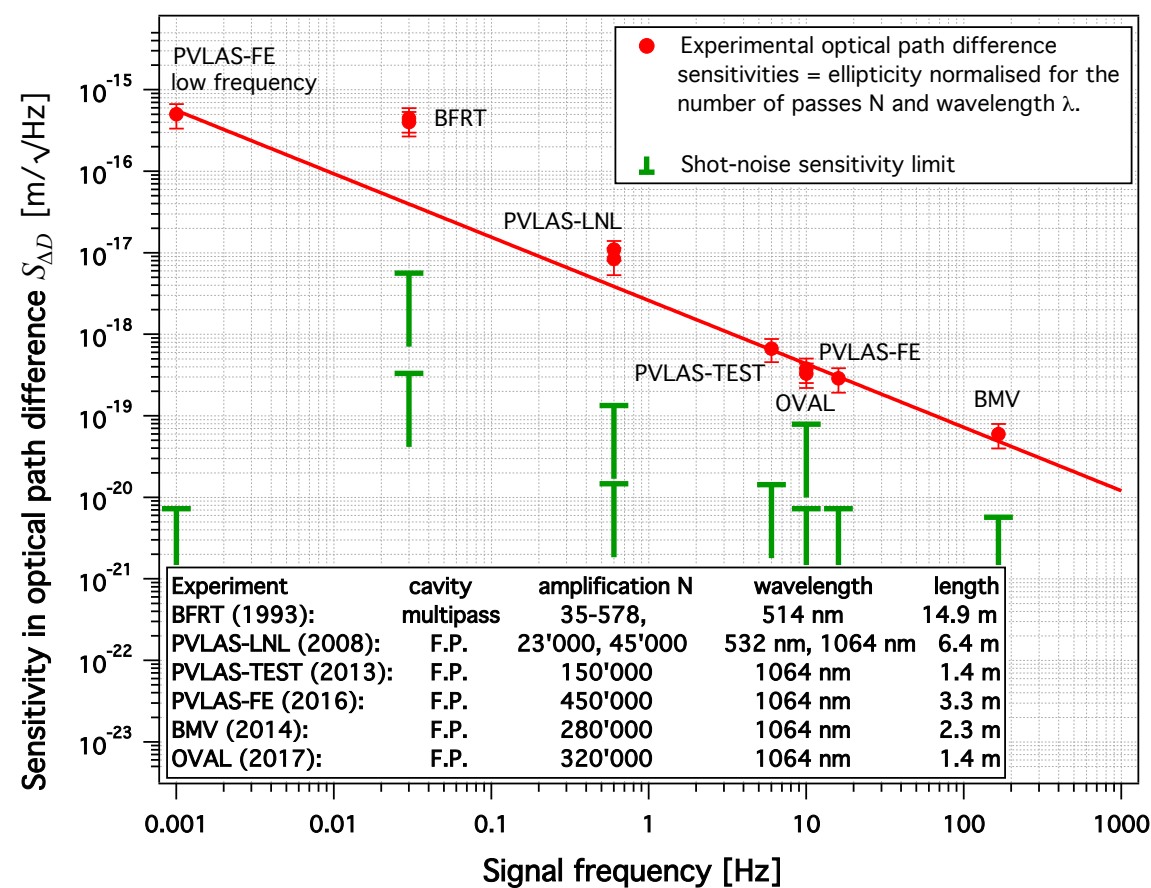

Figure 7. Measured optical path difference noise densities $S_{\Delta \mathcal{D}}$ in polarimeters set up to measure VMB plotted as a function of their signal frequency. Data were taken from the experiments BFRT [40], PVLAS-LNL [44,45], PVLAS-Test [61], PVLAS-FE [59], BMV [62] and OVAL [63]. The error bars are an estimated $50 \%$.

A more accurate study performed on the PVLAS-FE apparatus in Ferrara resulted in a good fit of $S_{\Delta \mathcal{D}}$ with a function proportional to $v^{-1 / 2}$ at low frequencies, with a cutoff at $v_{0}=15 \mathrm{~Hz}$, and proportional to $v^{-1 / 4}$ above $v_{0}$ :

$$
S_{\Delta \mathcal{D}}^{(\mathrm{fit})}(v)=\sqrt{\left(\frac{A_{\mathrm{th}} v^{-1 / 2}}{\sqrt{1+\left(v / \nu_{0}\right)^{2}}}\right)^{2}+\left(B_{\mathrm{th}} v^{-1 / 4}\right)^{2}}
$$

where one finds $A_{\text {th }}=(2.01 \pm 0.02) \times 10^{-18} \mathrm{~m}, v_{0}=(15.0 \pm 0.4) \mathrm{Hz}$ and $B_{\text {th }}=(4.63 \pm$ $0.02) \times 10^{-19} \mathrm{~m} / \mathrm{Hz}^{1 / 4}$. These noise contributions to $S_{\Delta \mathcal{D}}$ seem compatible to having an intrinsic thermal origin: $A_{\text {th }}$ we believe has a Brownian noise origin whereas $B_{\text {th }}$ is due to the photo-elastic effect in the mirror coating [1].

Finally, in the absence of the Fabry-Perot, shot-noise is achieved at output powers $I_{\|} \approx 10 \mathrm{~mW}$ resulting in $S_{\Psi}=5 \times 10^{-9} / \sqrt{\mathrm{Hz}}$ corresponding to $S_{\Delta \mathcal{D}}=1.6 \times 10^{-15} \mathrm{~m} / \sqrt{\mathrm{Hz}}$.

These studies have shown that an apparatus designed to measure VMB must be equipped with a magnetic field with $\int_{L_{B}} B_{\text {ext }}^{2} d L$ such that

$$
3 A_{e} \int_{L_{B}} B_{\text {ext }}^{2} d L>\frac{S_{\Delta \mathcal{D}}(v)^{(\text {fit })}}{\sqrt{\mathcal{T}}}
$$

where $S_{\Delta \mathcal{D}}(v)^{(\text {fit })}$ is taken from Equation (58) and $\mathcal{T}$ is the integration time.

\subsection{Calibration}

Two quantities need to be extracted during calibration measurements: the correction factor $k\left(\delta_{\mathrm{EQ}}\right)$ due to the birefringence of the cavity and the absolute amplitude and phase 
calibration. Magnetic birefringence in gases, known as the Cotton-Mouton effect [64], is used to determine this information: as discussed in Section 2.1 the ratio of the values of the 'spurious' rotation to ellipticity of Equation (46) gives $\delta_{\mathrm{EQ}}$; the observed ellipticity, corrected for $k\left(\delta_{\mathrm{EQ}}\right)$, defines an absolute scale for the magneto-optical effects. Once the absolute scale has been calibrated, $\delta_{\mathrm{EQ}}$ can be continuously monitored by inducing a Faraday effect on one of the mirrors and this time comparing the 'spurious' ellipticity to the 'true' rotation: $\left|\Delta_{\mathrm{EQ}} / 2\right|=\left|\Psi_{\text {(spurious) }} / \Phi\right|$.

The Cotton-Mouton - or Voigt - effect is perfectly analogous to VMB also depending on $\int_{L_{B}} B_{\text {ext }}^{2} d L$ but is far more intense already at low gas pressures. The birefringence generated in a gas at pressure $P$ by a magnetic field $B_{\text {ext }}$ is given by the expression

$$
\Delta n=n_{\|}-n_{\perp}=\Delta n_{u} B_{\mathrm{ext}}^{2} P
$$

where $\Delta n_{u}$ is the unit birefringence generated by $P=1 \mathrm{~atm}$ and $B_{\mathrm{ext}}=1 \mathrm{~T}$. In Table 1 we report some values of $\Delta n_{u}$ including the equivalent partial pressure $P_{\mathrm{EQ}}$ which would induce a birefringence equal to VMB. Other molecules and older values, also at different wavelengths, for the species listed here can be found in [64].

Table 1. Unitary magnetic birefringence of common inorganic gaseous species. The equivalent partial pressure which would mimic a VMB signal are also reported.

\begin{tabular}{ccccc}
\hline Species & $\boldsymbol{\Delta} \boldsymbol{n}_{\boldsymbol{u}}\left(\mathbf{T}^{-2} \mathbf{a t m} \mathbf{1}^{-1}\right)$ & $\lambda(\mathbf{n m})$ & $\boldsymbol{P}_{\mathrm{EQ}}(\mathbf{m b a r})$ & Ref. \\
\hline $\mathrm{He}$ & $(2.08 \pm 0.14) 10^{-16}$ & 1064 & $1.9 \times 10^{-5}$ & {$[65]$} \\
\hline & $(2.22 \pm 0.16) 10^{-16}$ & 1064 & $1.8 \times 10^{-5}$ & {$[66]$} \\
\hline $\mathrm{Ne}$ & $(2.7 \pm 0.3) 10^{-16}$ & 1064 & $1.5 \times 10^{-5}$ & {$[67]$} \\
\hline $\mathrm{Ar}$ & $(6.9 \pm 0.2) 10^{-16}$ & 1064 & $5.8 \times 10^{-6}$ & {$[68,69]$} \\
\hline & $(4.5 \pm 0.5) 10^{-15}$ & 1064 & $5.3 \times 10^{-7}$ & {$[59]$} \\
\hline $\mathrm{H}_{2} \mathrm{O}$ & $(6.67 \pm 0.38) 10^{-15}$ & 1064 & $9.3 \times 10^{-7}$ & {$[70]$} \\
\hline $\mathrm{H}_{2}$ & $(8.28 \pm 0.57) 10^{-15}$ & 1064 & $6.0 \times 10^{-7}$ & {$[71]$} \\
\hline $\mathrm{CH}_{4}$ & $(1.59 \pm 0.21) 10^{-14}$ & 514 & $4.8 \times 10^{-7}$ & {$[64]$} \\
\hline $\mathrm{Kr}$ & $(9.98 \pm 0.40) 10^{-15}$ & 1064 & $2.5 \times 10^{-7}$ & {$[72]$} \\
\hline $\mathrm{Xe}$ & $(2.85 \pm 0.25) 10^{-14}$ & 1064 & $1.4 \times 10^{-7}$ & {$[69,73]$} \\
\hline & $(2.59 \pm 0.40) 10^{-14}$ & 1064 & $1.5 \times 10^{-7}$ & {$[69,73]$} \\
\hline $\mathrm{C}_{2} \mathrm{H}_{6}$ & $(-1.48 \pm 0.09) 10^{-13}$ & 632.8 & $2.7 \times 10^{-8}$ & {$[70]$} \\
\hline $\mathrm{CO}$ & $(-1.80 \pm 0.06) 10^{-13}$ & 546 & $2.2 \times 10^{-8}$ & {$[64]$} \\
\hline $\mathrm{N}_{2}$ & $-(2.66 \pm 0.42) 10^{-13}$ & 1064 & $1.5 \times 10^{-8}$ & {$[70,75]$} \\
\hline $\mathrm{CO}_{2}$ & $(-4.22 \pm 0.31) 10^{-13}$ & 1064 & $9.5 \times 10^{-9}$ & {$[70]$} \\
\hline $\mathrm{O}_{2}$ & $(-2.29 \pm 0.08) 10^{-12}$ & 1064 & $1.8 \times 10^{-9}$ & {$[76]$} \\
\hline
\end{tabular}

The phase of the Cotton-Mouton effect (including its sign) defines the physical phase for a field induced ellipticity: VMB must come with the same phase as the Cotton-Mouton measurement of a noble gas with $\Delta n_{u}>0$. When analyzing data, all the measured signals are projected onto both the physical and the non-physical axes. We explicitly note that the gas measurements are interpreted in terms of a pure birefringence. In fact, for gases, no linear dichroism is associated with a transverse magnetic field. A Faraday rotation resulting from a time variation of an eventual small longitudinal component of the magnetic field along the light path would appear at the first harmonics of the rotation frequency of the magnetic field. 


\section{Measuring Vacuum Magnetic Birefringence and Searching for ALPs in Italy:} The PVLAS Collaboration

\subsection{PVLAS-LNL: Rotating Superconducting Magnet}

\subsubsection{Infrastructure}

Earlier experience from precursor experiments led to a first serious effort to measure VMB using the scheme in Figure 5. A first version of the experiment PVLAS (Polarizzazione del Vuoto con LASer) financed by INFN (Istituto Nazionale di Fisica Nucleare) was installed at the INFN-Laboratori Nazionali di Legnaro near Padova, Italy (referred to as PVLASLNL from now on). Two major changes were introduced with respect to the precursor tests to improve the expected sensitivity: the use of a vertically rotating superconducting magnet (in persistent current mode) and of a Fabry-Perot resonant cavity. The cavity length was $6.4 \mathrm{~m}$ and the rotating field allowed signal frequencies at $2 v_{B} \approx 0.6 \mathrm{~Hz}$, a frequency a factor 30 higher with respect to current modulated superconducting magnets. The maximum field used was limited to $B_{\text {ext }}=5.5 \mathrm{~T}$ with a length of $L_{B}=1.05 \mathrm{~m}$. Runs with various field intensities were used resulting in $B_{\text {ext }}^{2} L_{B}=(5 \div 30) \mathrm{T}^{2} \mathrm{~m}$.

A drawing and a photograph of the setup are shown in Figure 8. A dedicated infrastructure was designed and constructed at LNL to install the superconducting dipole magnet and its rotating cryostat in a vertical configuration. To avoid mechanical vibrations coming from the rotating magnet to reach the optical benches the experimental hall was equipped with a square pit, $8 \times 8 \mathrm{~m}^{2}, 3 \mathrm{~m}$ deep from the floor level of the main building. A concrete 'raft', formed the floor of the pit and was supported by pillars embedded $14 \mathrm{~m}$ deep into sand. The lower optical bench, the $7 \mathrm{~m}$ high vertical structure and the upper optical bench, all made of black granite sat on the 'raft'. A reinforced concrete beam, crossing over the pit and sitting on the floor of the main building, supported the rotating table driven by a hydraulic motor on which sat the cryostat containing the magnet. In this way, no direct mechanical contact was present between the rotating cryostat and the vertical optical structure. In the photograph of Figure 8 the ladder leaning against the structure is standing on the concrete beam supporting the rotating turntable. During the rotation of the magnet no ellipticity or rotation signal was observed correlated with the non-energized magnet rotation.
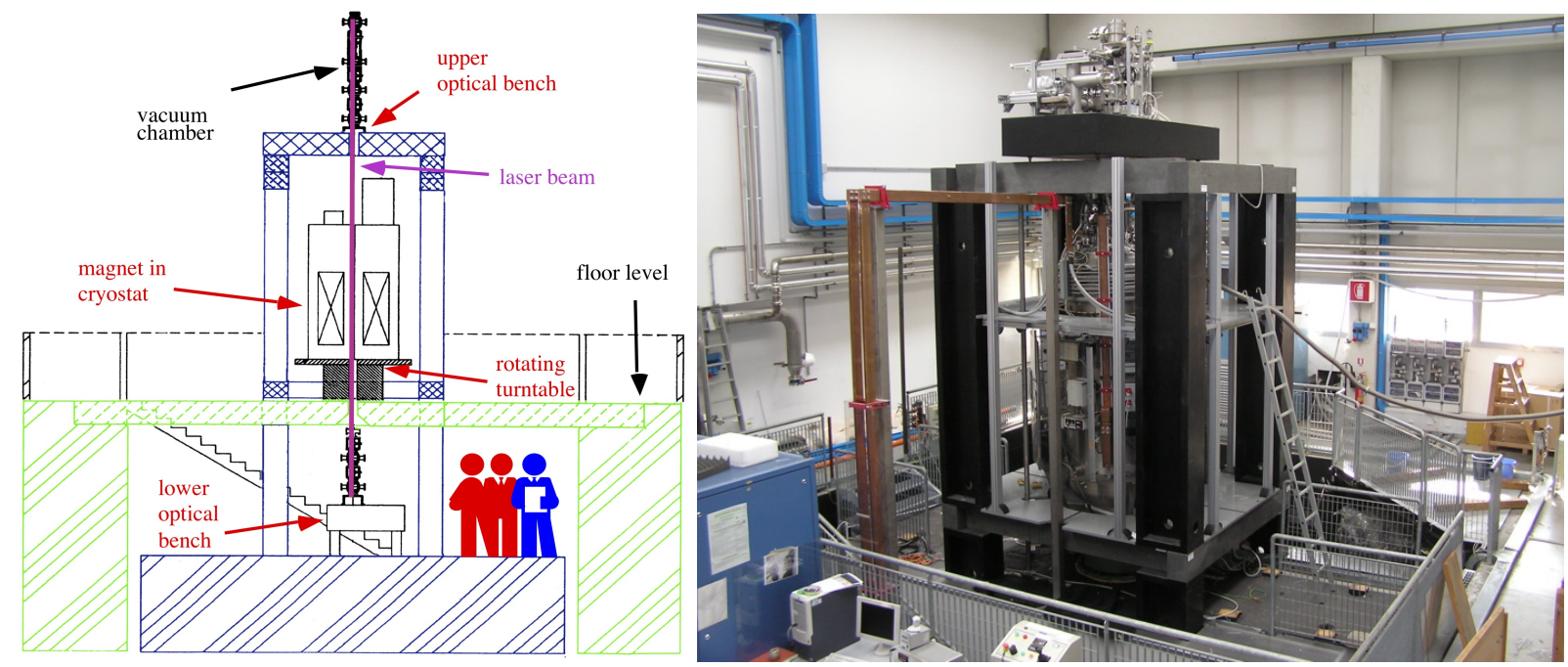

Figure 8. (Left) schematic drawing of the PVLAS-LNL setup. (Right) a photograph of the apparatus above the floor level. The lower optical bench is below the beam supporting the cryostat and sits about $3 \mathrm{~m}$ below ground level on a concrete 'raft'.

\subsubsection{Rotating Superconducting Magnet}

The magnet used in this phase was the original $1 \mathrm{~m}$ long superconducting dipole magnet developed by Mario Morpurgo at CERN [77] as a prototype for the CERN D2 Proposal $[78,79]$, the first proposal to measure VMB using the optical technique proposed a 
couple of years before [52]. It was manufactured at CERN and commissioned in July 1982 and at the time reached a record field of $8.8 \mathrm{~T}$ when cooled with superfluid liquid helium. Its main characteristics are listed in Table 2.

Table 2. Main characteristics of the PVLAS-LNL magnet built at CERN by Mario Morpurgo as a prototype for the original D2 CERN proposal to measure VMB.

\begin{tabular}{cc}
\hline Cold bore useful diameter & $0.1 \mathrm{~m}$ \\
\hline Magnetic field length & $1 \mathrm{~m}$ \\
\hline Overall magnet length & $1.3 \mathrm{~m}$ \\
\hline Current (corresponding to a central field of $8 \mathrm{~T}$ ) & $3810 \mathrm{~A}$ \\
\hline Max field on the conductor & $8.8 \mathrm{~T}$ \\
\hline Field uniformity in the useful bore & $\pm 2.5 \%$ \\
\hline Average current density in the winding & $105 \mathrm{~A} / \mathrm{mm}^{2}$ \\
\hline Stored energy & $1.5 \mathrm{MJ}$ \\
\hline
\end{tabular}

The 2.3-ton dipole magnet was wound with a hollow $\mathrm{Cu}-\mathrm{Nb}$-Ti composite conductor. The conductor had a square cross-section $5.5 \times 5.5 \mathrm{~mm}^{2}$ with a central bore approximately $2.5 \mathrm{~mm}$ diameter for cooling. The dipole was composed of two identical coils each with 12 pancakes of 22 turns each. The 12 pancakes were subdivided into three groups of four, and each group was separately impregnated under vacuum with epoxy resin. The dipole yoke was made from soft iron. Interestingly, aluminum alloy plates and bolts were used to clamp together the various parts of the yoke. Since the aluminum thermal contraction is larger compared to the other metals, the coils were strongly compressed when cooled guaranteeing mechanical stability.

In Figure 8, right, the solid copper bars connecting the magnet to the $10 \mathrm{~V}, 5000 \mathrm{~A}$ power supply (blue cabinet on the left) can be seen. Inside the power supply a set of watercooled diodes and resistors were installed to dump the electromagnetic energy stored during the (fast) discharge. Once charged, the magnet was put in 'quasi persistent' current mode through a very low resistance $(0.24 \mu \Omega)$ silver-plated copper multi-blade socket [80] and the power supply disconnected.

\subsubsection{Rotating Cryostat}

The project of the cryostat was realized at LNL in collaboration with CERN [81] and was designed to cool the magnet to superfluid liquid helium temperature. It was characterized by a room temperature $30.5 \mathrm{~mm}$ central bore, to host the quartz vacuum tube through which the light beam traverses the magnet, and by the possibility to rotate around its axis.

The scheme of the cryostat, shown in Figure 9, is of the Claudet type [82] with a fiber glass epoxy 'lambda' plate to separate the superfluid helium at the bottom from the normal helium above. The outer jacket was superinsulated with mattresses of superinsulation. At the bottom of the cryostat and at its center, a few kilograms of activated charcoal were placed, to lower the pressure in the superinsulation region, thus improving the necessary insulation efficiency. Multi-layer superinsulation was also used for the central bore. Inside the cryostat a stainless steel coil was wound around the magnet as a heat exchanger for the pre-cooling with liquid nitrogen (LN2) using helium gas as an exchange medium.

Vacuum pumps for the superfluid helium production were installed on platforms outside of the cryostat and rotated with it. These were never used during the measurements due to a limited availability of helium, hence we never reached the maximum field. The helium boil-off vapors were collected at room temperature, both with the cryostat at rest and during the rotation, through a helium tight feedthrough and sent to the recovery system. 


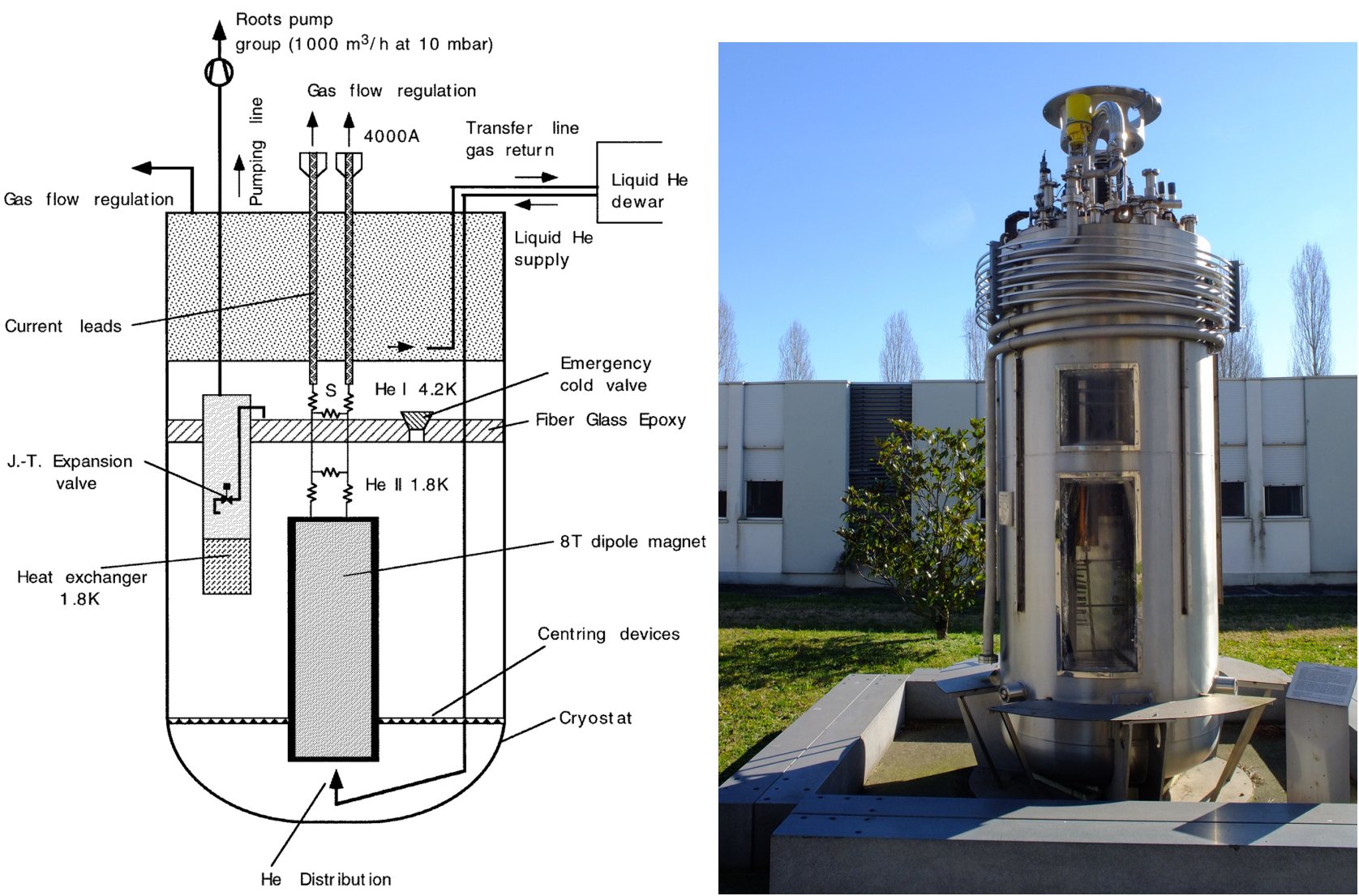

Figure 9. (Left) Scheme of the rotating cryostat of the PVLAS-LNL experiment designed to cool the dipole magnet to superfluid helium. A $30.5 \mathrm{~mm}$ warm bore (not shown) vertically traversed the cryostat. Source: From Ref. [83], (C) IOP Publishing. Reproduced with permission. All rights reserved). (Right) Photograph of the cryostat of the PVLAS-LNL experiment. The cryostat is exhibited on the INFN National Laboratories of Legnaro (LNL) site near Padua, Italy. In the cutaway the prototype CERN magnet can be seen.

In the PVLAS-LNL configuration the limiting factor on integration time and more importantly on the capability of debugging for systematics was the availability of liquid helium.

\subsubsection{Polarimeter Setup and Results}

Figure 10 is a schematic drawing of the optical setup of PVLAS-LNL for ellipticity measurements (QWP not inserted). A Nd:YAG laser is frequency locked to the Fabry-Perot cavity using a modified Pound-Drever-Hall technique. Two wavelengths were used during the working period of the experiment: $1064 \mathrm{~nm}$ and $532 \mathrm{~nm}$. At $1064 \mathrm{~nm}$ the finesse of the cavity was about $\mathcal{F}^{(1064)}=70,000$ whereas at $532 \mathrm{~nm}$ it was about $\mathcal{F}^{(532)}=35,000$. The beam then follows the scheme of Figure 5. As the ellipticity modulator first a $20 \mathrm{kHz}$ PEM was used and in the final version of the PVLAS-LNL setup the home-made SOM was used allowing the control of $\gamma_{\mathrm{DC}}$.

As discussed above the signal from the photodiode is amplified and demodulated at $v_{m}$ and $2 v_{m}$ (only one lock-in is shown). The DAQ is triggered by the turntable with 32 equally spaced marks: independently of the slight frequency variations of the hydraulic drive, the phase-preserving appending of different data sets gave long integration times. The typical rotation frequency of the cryostat was $v_{B}=0.3 \mathrm{~Hz}$.

In the PVLAS-LNL configuration the relative intensity noise at $v_{m}$ was $N_{v}^{(\mathrm{RIN})} \approx 2 \times$ $10^{-5} / \sqrt{\mathrm{Hz}}$ and was the main limiting factor in the predicted ellipticity sensitivity: from Equation (54) one finds $S_{\Psi} \approx 1.5 \times 10^{-8} / \sqrt{\mathrm{Hz}}$. As already discussed in Section 2.3 the measured sensitivity was far from the expected value resulting in $S_{\Psi}^{(P V L A S-L N L)} \approx 10^{-6} / \sqrt{\mathrm{Hz}} @$ 
$0.6 \mathrm{~Hz}$ [45] both at $1064 \mathrm{~nm}$ and $532 \mathrm{~nm}$ which translated in optical path difference sensitivity as $S_{\Delta \mathcal{D}} \approx 10^{-17} \mathrm{~m} / \sqrt{\mathrm{Hz}} @ 0.6 \mathrm{~Hz}$.

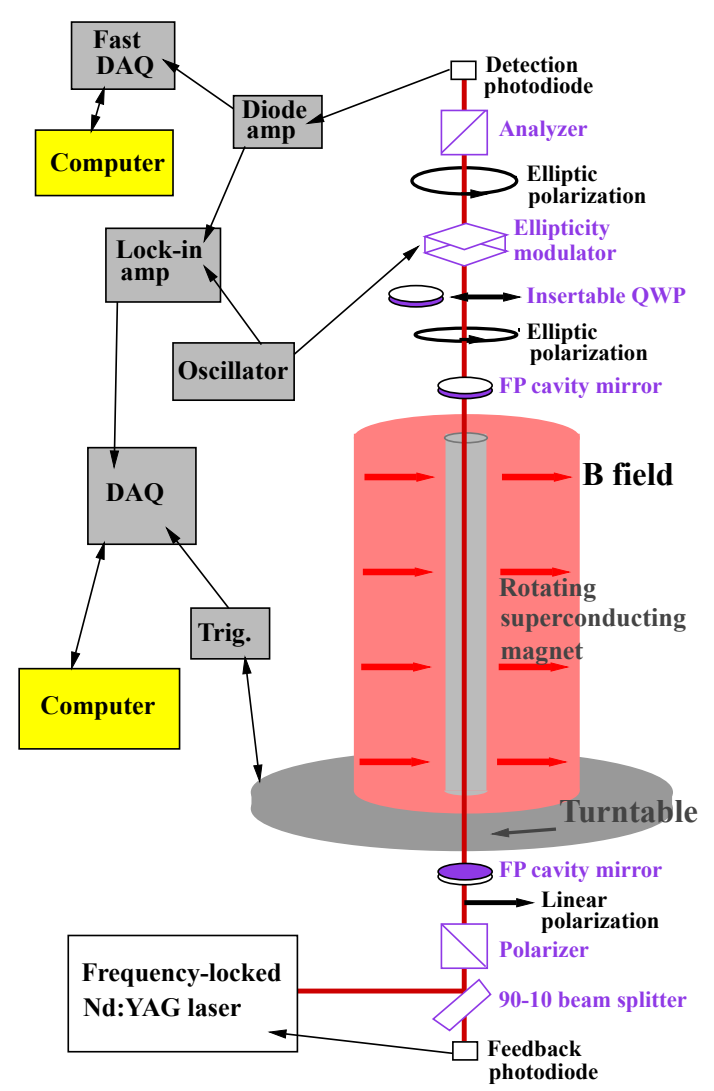

Figure 10. Schematic drawing of the PVLAS-LNL optical and acquisition setup.

During the time in which the experiment was active, PVLAS-LNL set the best limits of $\mathrm{VMB}$ at $95 \%$ c.l. [45]:

$$
\begin{aligned}
\Delta n^{(\text {PVLAS-LNL })} & <1.0 \times 10^{-19} \quad @ B=2.3 \mathrm{~T} \\
\left(\frac{\Delta n}{B_{\text {ext }}^{2}}\right)^{(\text {PVLAS-LNL })} & <1.9 \times 10^{-20} \mathrm{~T}^{-2} .
\end{aligned}
$$

Interestingly, this limit was obtained with a field strength $B=2.3 \mathrm{~T}$. Indeed at $B=5.5 \mathrm{~T}$ systematic effects limited ellipticity measurements. For rotation measurements instead the best limit was obtained with $B=5.0 \mathrm{~T}$ [44]:

$$
|\Delta \kappa|^{(\text {PVLAS-LNL })}<0.9 \times 10^{-19} \quad @ B=5.0 \mathrm{~T} .
$$

This limit on $|\Delta \kappa|$ corresponds to a difference in the absorption coefficients for the two orthogonal polarizations of $\Delta \mu<1.1 \times 10^{-12} \mathrm{~m}^{-1}$.

\subsection{PVLAS-FE: Rotating Permanent Magnets}

The evolution of the PVLAS-LNL apparatus was the result of three main requirements: (1) reduce seismic noise by installing the whole polarimeter on a single isolated optical bench as demonstrated in [84]; (2) use a two magnet configuration to ensure zero measurements with the magnetic field $\mathrm{ON}$ for efficient debugging of systematic effects due to the magnetic field [61] and compatible with a benchtop apparatus; (3) use magnets with an unlimited duty-cycle even with a slightly lower field with respect to achievable values. These considerations led to the PVLAS-FE setup shown in Figure 11. 
The apparatus is basically composed of a seismically isolated 4 ton granite optical bench hosting the optics of the polarimeter and vacuum system and of two permanent rotating $2.5 \mathrm{~T}$ dipole magnets each about 1 meter in length (mechanically) with a $2 \mathrm{~cm}$ axial bore.

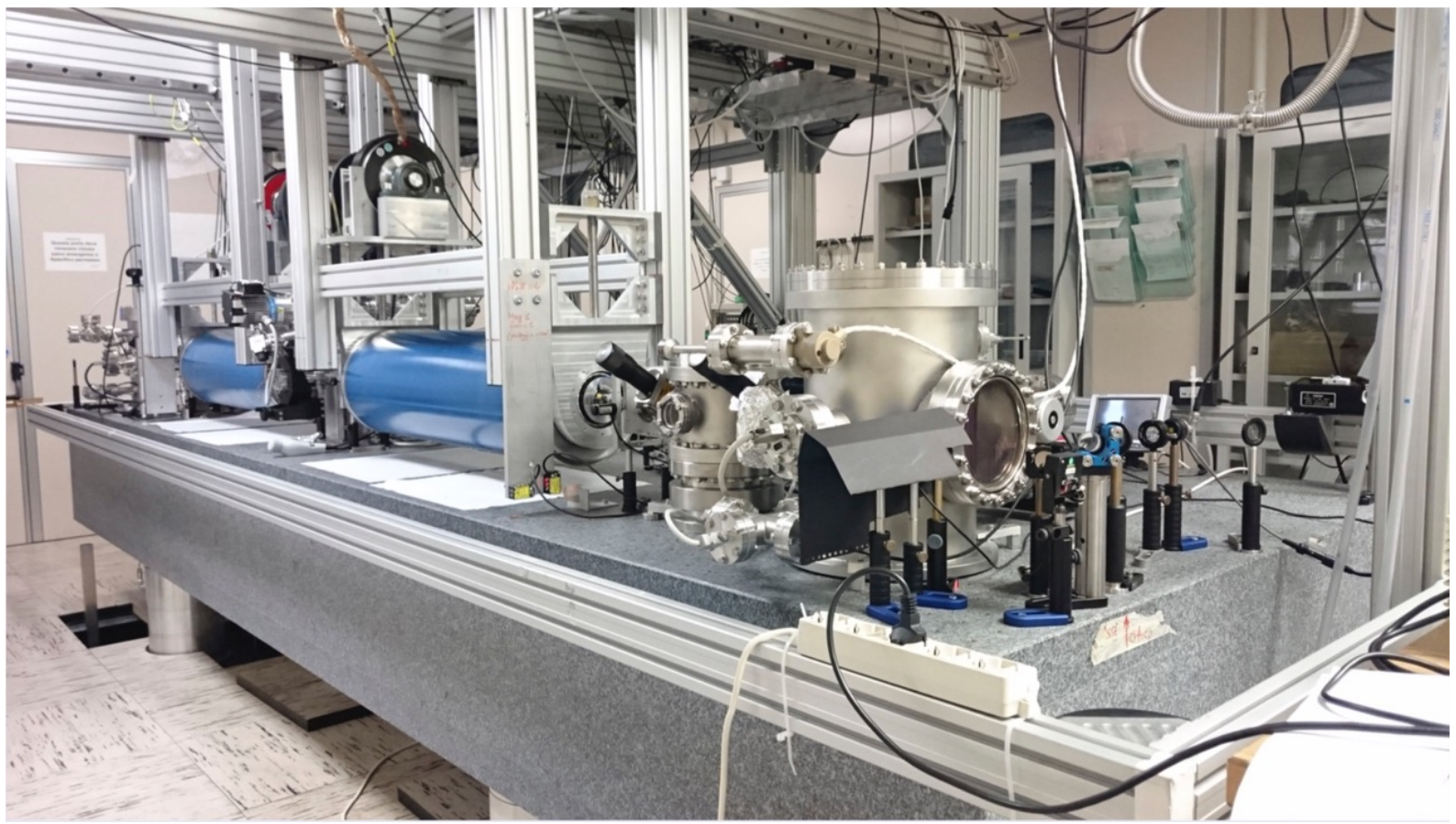

Figure 11. Photograph of the optical bench layout of the PVLAS-FE apparatus installed at the Department of Physics and Earth Sciences of the University of Ferrara and INFN Section of Ferrara, Ferrara, in Italy.

\subsubsection{Infrastructure, Optical Bench and Vacuum System}

The experiment is located on the ground floor of an experimental hall at the Department of Physics and Earth Sciences of the University of Ferrara and INFN Section of Ferrara, Ferrara, Italy, inside a temperature controlled $\left(23^{\circ} \pm 1^{\circ}\right)$ and relative humidity controlled $(\approx 56 \%)$ clean room of ISO-4 class.

As with what was done in PVLAS-LNL special care was taken to limit direct mechanical contact between the optical bench and the magnet support structure: the magnets were sustained by an aluminum structure sitting on the concrete floor whereas the optical bench was supported by a seismic isolation system (BiAir ${ }^{\circledR}$ membrane air spring legs) equipped with a six-degrees of freedom positioning system to guarantee a $10 \mu \mathrm{m}$ position repeatability of the bench with respect to the magnets. This was fundamental in that the vacuum tube passing through the bore of the two magnets was made of glass. From the specifications of the manufacturer (Bilz Vibration Technology AG, Leonberg, Germany) the filtering of the supporting system started at about $1 \mathrm{~Hz}$. The two magnets were independently driven by synchronous brushless motors to allow long in-phase integration.

Although the permanent magnets were designed following the Halbach configuration [85], which in principle cancels stray fields, a small stray field of about $10 \mathrm{mT}$ was present near their surface, rapidly decaying with distance. To avoid mechanical forces generated by the rotating stray field on nearby components granite was chosen as the bench material. The bench, manufactured by Microplan, Quarona (VC), Italy, is $4.8 \mathrm{~m}$ long, $1.5 \mathrm{~m}$ wide and $0.5 \mathrm{~m}$ thick for a total weight of 4 tons. A granite 'honeycomb' structure fills the inside of the bench to limit the total weight. The surface of the bench is equipped with a $5 \times 5 \mathrm{~cm}$ matrix of threaded holes made of brass.

The light propagates in vacuum from before the entrance polarizer to after the analyzer. The vacuum system, completely constructed in nonmagnetic materials, is divided in two larger vacuum chambers containing the entrance and output polarizing optics, two smaller 
chambers with isolation valves containing the Fabry-Perot mirrors and a glass vacuum tube passing through the two magnets. Between the two magnets is an auxiliary pumping station. Only dry pumps were used, and the isolation valves of the mirror chambers guaranteed the stability of the extremely high finesse for about 5 years with the same pair of mirrors (until the mirrors were dismounted).

Initially vacuum is guaranteed by turbo molecular pumps connected to the two larger chambers (see Figure 11) and to the central auxiliary station. After reaching a vacuum pressure $\approx 10^{-7}$ mbar three non-evaporable getter (NEG) pumps, connected to the same chambers as the turbo pumps, are opened and the turbo pumps in the large chambers are switched off to limit vibrations reaching the optics. The central turbo pump needs to be left on to pump away the noble gases which the NEG pumps do not pump. Ionic pumps were avoided due to the presence of a magnet.

This system guaranteed a stable vacuum of $\approx 2 \times 10^{-8}$ mbar in the three pumping chambers with a typical residual gas mass spectrum shown in Figure 12.

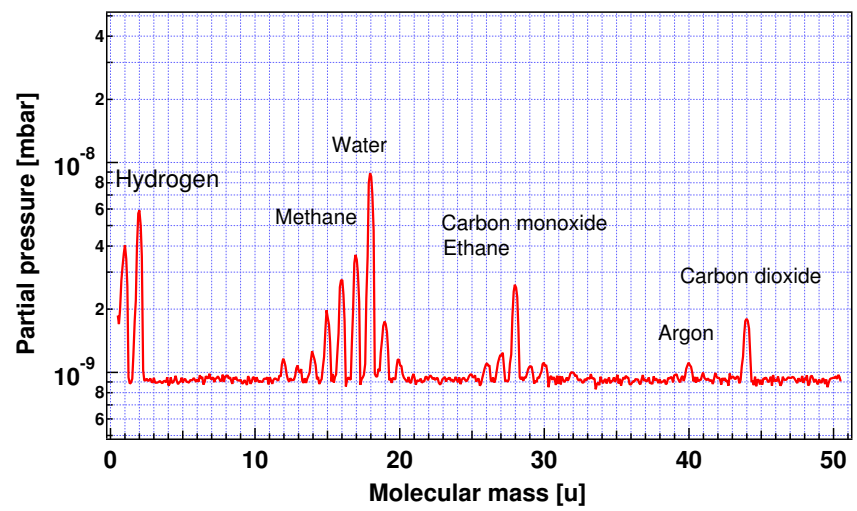

Figure 12. Typical residual gas mass spectrum during data taking, measured in the large output chamber. The main residual gases (from left to right) are hydrogen, methane, water vapor, carbon monoxide, and carbon dioxide. The absence of the oxygen peak indicates the absence of nitrogen. All gas pressures were well below the VMB equivalent pressures reported in Table 1 on page 15.

\subsubsection{The Rotating Permanent Magnets}

The permanent magnets of the PVLAS-FE experiment take advantage of the recently developed Nd-Fe-B sintered magnet technology and were built by AMT\&C (Moscow, Russia). The set up comprises two identical dipole magnets of the Halbach type [85], with $B=2.5 \mathrm{~T}$. The main technical characteristics of each magnet are listed in Table 3 .

Table 3. Main characteristics of the dipole magnets designed and built by Advanced Magnetic Technologies \& Consulting LLC, Troitsk, Russian Federation.

\begin{tabular}{cc}
\hline Magnetic system design & cylindrical \\
\hline Magnetic field direction & normal to bore axis \\
\hline Overall length & $934 \mathrm{~mm}$ \\
\hline Outer diameter & $280 \mathrm{~mm}$ \\
\hline Bore diameter & $20 \mathrm{~mm}$ \\
\hline Net weight & $450 \mathrm{~kg}$ \\
\hline Magnetic material & high coercivity Nd-Fe-B \\
\hline Maximum field intensity $B_{\text {ext }}$ & $2.5 \mathrm{~T}$ \\
\hline Squared field integral & $5.12 \mathrm{~T}^{2} \mathrm{~m}$ \\
\hline Magnetic field length $L_{B}$ & $0.82 \mathrm{~m}$ \\
\hline
\end{tabular}


The segments of the Halbach structure were divided into two concentric rings. Each ring was composed of 16 sectors of $\mathrm{Nd}-\mathrm{Fe}-\mathrm{B}$ pre-magnetized material. In Figure 13 one can see a drawing of the concentric structure of the PVLAS-FE magnets and a theoretical magnetization direction of the various sectors. Twelve layers each $70 \mathrm{~mm}$ thick were assembled axially in a cylindrical soft magnetic steel case. To minimize the stray field on the mirrors and polarizing optics each end flange was a four-layer stack of alternating aluminum and steel. Each magnet was balanced by the manufacturer according to $G 2.5$ accuracy class ISO 1940-1. The measured transverse component of the magnetic field for the two magnets is shown in Figure 14.
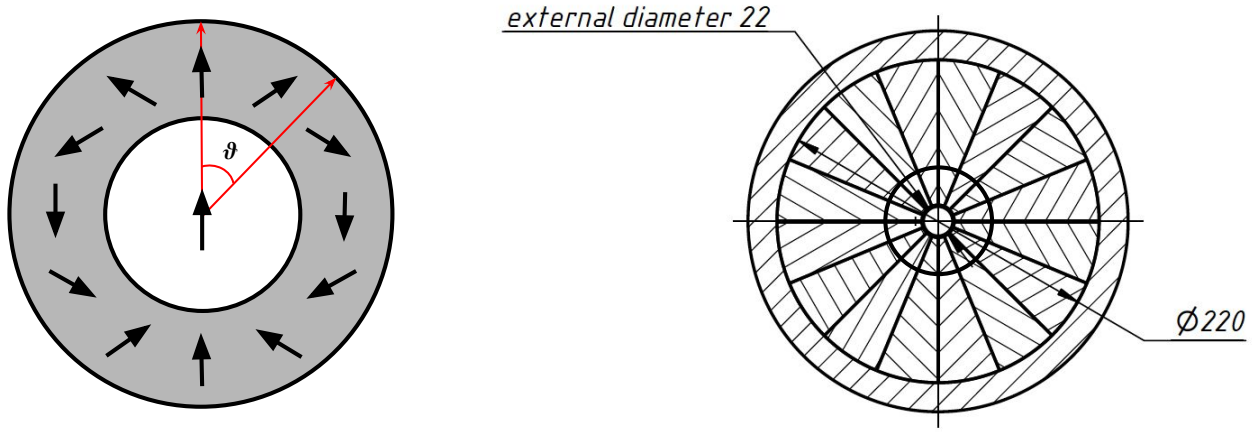

Figure 13. (Left) Magnetization directions in the Halbach configuration of a dipole field. (Right) Two rings, 16 segment Halbach configuration of the PVLAS-FE magnets with the external cylindrical enclosure.
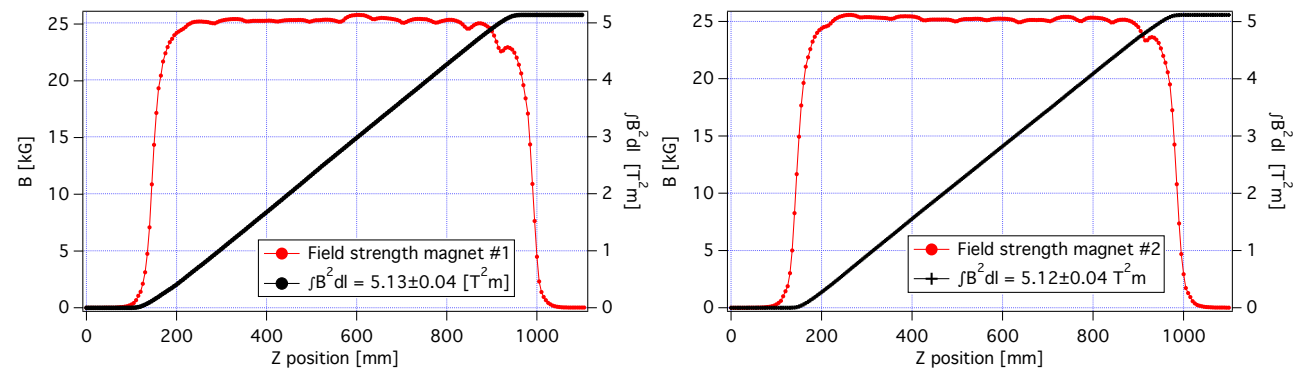

Figure 14. In red are the measured dipolar field profiles of the two PVLAS-FE magnets. The values of $\int B_{\mathrm{ext}}^{2} d L$ are reported in black.

As already mentioned, the magnets were supported by an aluminum nonmagnetic structure set on the concrete floor of the experimental hall. There is no direct contact between the optical bench and the magnet support structure. The support structure allows the horizontal movement of the magnets for their extraction and their orientation for optimal alignment with the glass vacuum tubes: two different glass tubes with diameters $18 \mathrm{~mm}$ and $15 \mathrm{~mm}$ were used whereas the magnet bores have a diameter of $20 \mathrm{~mm}$. The two magnets are kept in rotation by V-toothed low vibration belt transmissions driven by two independent brushless motors whose rotation frequencies is determined by phaselocked independent signal generators. In this way, the rotation of the magnets is controlled in phase: during data taking the angular position of the magnetic fields was exactly known at any time. To allow for systematics monitoring and debugging, the two magnets were generally rotated at two slightly different frequencies.

\subsubsection{Optical Setup}

The scheme of the optical setup of the apparatus is shown in Figure 15. All optical mounts and motorized stages are nonmagnetic and are based on the 'slip-stick' principle (SmarAct GmbH, Oldenburg, Germany) ensuring stable positions even with the power 
switched off. A $\lambda=1064 \mathrm{~nm}$ laser beam is emitted by a Nd:YAG laser (Innolight Mephisto, $2 \mathrm{~W}$ power). The beam first passes through a quarter-wave plate (QWP), a half-wave plate (HWP) and a two stage Faraday isolator to reduce the initial ellipticity of the laser beam, to allow power adjustment and to block back-reflected light from entering the laser. The beam then passes through a mode matching lens for optimal coupling of the laser to the Fabry-Perot cavity. Two steering mirrors followed by a second HWP bring the beam to the entrance of the vacuum system with the desired alignment and polarization direction. Between the second steering mirror and this second HWP a beam sampler of the reflected power from the cavity is used for phase-locking the laser to the cavity via the Pound-Drever-Hall (PDH) technique. The same beam sampler is also used to sample the beam power at the Fabry-Perot input. The sidebands for the PDH locking circuit were generated directly in the laser rather than with an external phase modulator $[54,55]$. An automatic locking servo-circuit allowed operation of the apparatus with an almost unitary duty-cycle.

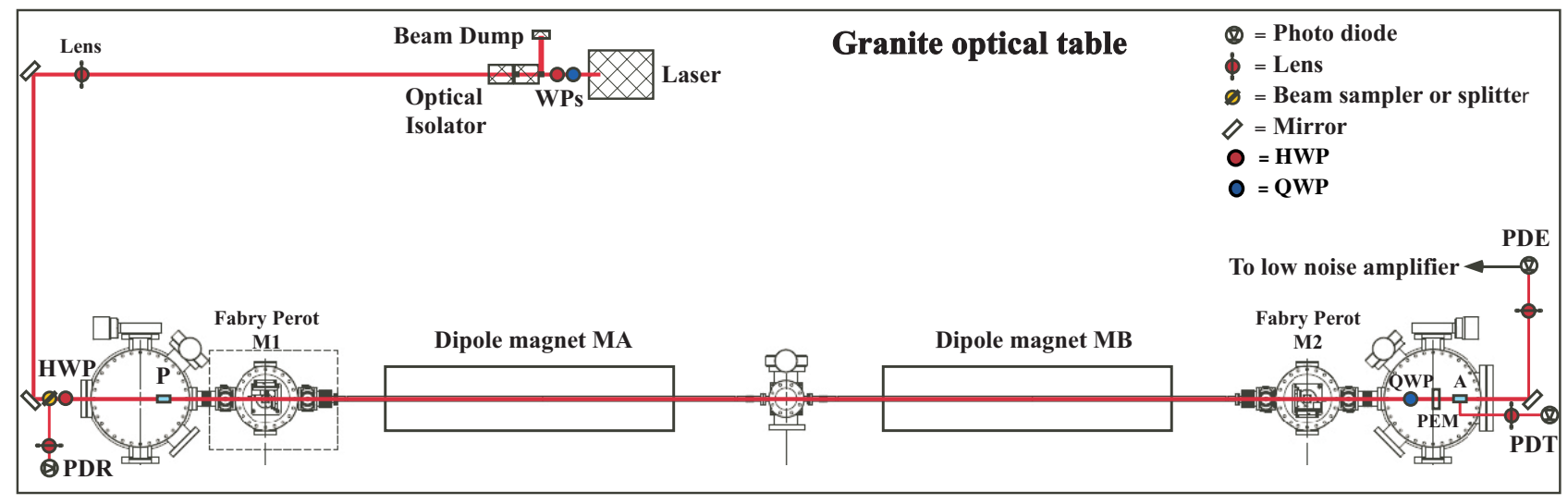

Figure 15. Schematic view of the optical bench layout of the PVLAS-FE apparatus. The laser beam enters the polarizing vacuum chamber (left) containing the polarizer P after having been properly aligned and focused. It then passes through the mirrors M1 and M2 of the Fabry-Perot and the magnets. The analyzing chamber (right) contains in order an extractable QWP (shown inserted) for rotation measurements, the PEM ellipticity modulator and the analyzer A with an extra lateral output. HWP = Half-wave plate; $\mathrm{QWP}=$ quarter-wave plate; $\mathrm{PDT}=$ transmission signal $I_{\|} ; \mathrm{PDE}=\operatorname{extinction}$ signal $I_{\perp}$.

The second HWP together with the rotatable polarizer $\mathrm{P}$ allows the alignment of the light polarization with one of the axes of the equivalent wave plate of the Fabry-Perot cavity before entering it. At the output of the cavity an extractable QWP (shown inserted in Figure 15) is used to transform, when necessary, a polarization rotation into an ellipticity (and vice versa). The light then passes through the $v_{m} \approx 50 \mathrm{kHz}$ resonant photo-elastic ellipticity modulator, PEM, (Hinds Instruments, Hillsboro, OR, USA), and the analyzer A, normally set to maximum extinction. The PEM is mounted on an axial rotation mount to set its axis at $45^{\circ}$ with respect to the polarization direction, and on a translation stage to allow its extraction from the beam. Typically, the modulation amplitude is between $\eta_{0} \approx 3 \times 10^{-3} \div 10^{-2}$ to work in the optimal modulation region shown in Figure 6. An exit window on the analyzer allows the extraction of both the extraordinary and ordinary beams: the former is a measurement of the power $I_{\|} \approx I_{\text {out }}$ transmitted by the cavity, whereas the extinguished beam power, $I_{\perp}$, contains the information on the ellipticity and rotation acquired by the light. The extinction ratio was generally $\sigma^{2} \lesssim 10^{-7}$. An InGaAs low noise photodiode with gain $G=10^{6} \mathrm{~V} / \mathrm{A}$ and efficiency $q=0.7 \mathrm{~A} / \mathrm{W}$ is used to collect the extinguished light. The diode was placed about $2 \mathrm{~m}$ from the analyzer to reduce contamination from diffused light.

It is worthwhile to note here that the glass vacuum tubes passing through the two rotating magnets were equipped with baffles to eliminate diffused light from the mirrors which was not part of the main Gaussian $\mathrm{TEM}_{00}$ mode. These baffles were O-rings spaced in such a way that from the position of the light spot on the mirrors none of the surface 
of the glass tube could be seen. With this expedient and with a careful monitoring of the movement of the glass tube with accelerometers a noise floor without the presence of systematic signals was obtained with a total composite integration of $\mathcal{T}=5 \times 10^{6} \mathrm{~s}$.

For completeness in Figure 16 we report the optical path difference noise in the PVLASFE polarimeter filled with $850 \pm 20 \mu \mathrm{bar}$ of Argon gas to generate reference ellipticity values. Two peaks at $8 \mathrm{~Hz}$ and $10 \mathrm{~Hz}$ of equal height can be seen due to the Cotton-Mouton effect of Argon. A peak at $19 \mathrm{~Hz}$ is also visible and is due to a Faraday rotation generated by a solenoid applied to the entrance mirror of the cavity which generates a small ellipticity due to the birefringence of the cavity according to Equation (43). Superimposed is the fit with Equation (58). Note that during VMB measurements, the noise is the same.

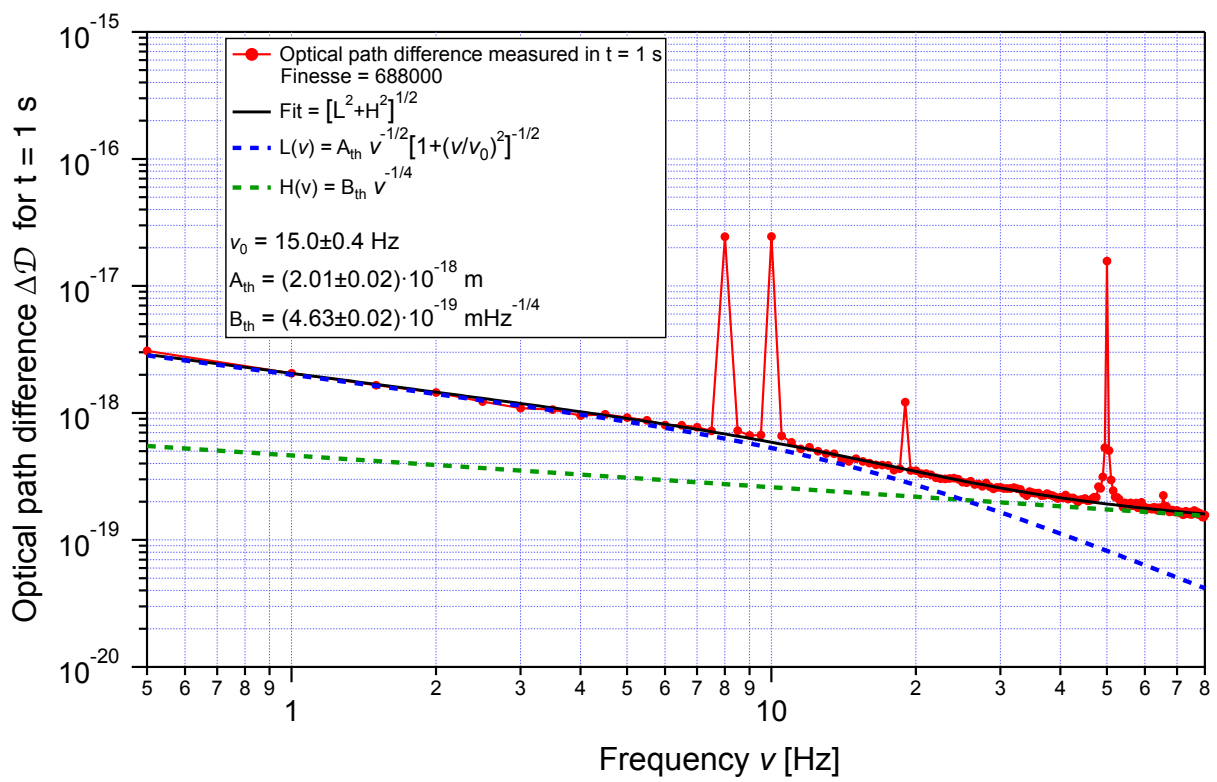

Figure 16. Optical path difference spectrum for $\mathcal{F}=688,000$ measured with the PVLAS-FE apparatus in $\mathrm{t}=1 \mathrm{~s}$ with $(850 \pm 20) \mu \mathrm{bar}$ of Ar generating the two identical peaks at $8 \mathrm{~Hz}$ and $10 \mathrm{~Hz}$. Superimposed is the fit obtained using Equation (58). The dashed lines are the two contributions to the curve describing the fit.

The final PVLAS-FE sensitivity in ellipticity was $S_{\Psi} \approx 5 \times 10^{-7} / \sqrt{\mathrm{Hz}}$ resulting in a notable sensitivity in optical path difference of $S_{\Delta \mathcal{D}} \approx 4 \times 10^{-19} \mathrm{~m} / \sqrt{\mathrm{Hz}}$ at $v=16 \mathrm{~Hz}$ (frequency used during long integration time measurements) again limited by the intrinsic birefringence noise of the cavity mirrors according to Equation (58).

\section{Results}

\subsection{VMB Measurements}

Although not sufficient to detect VMB, the PVLAS-FE apparatus set the present best limit in vacuum magnetic birefringence of

$$
\begin{aligned}
\Delta n^{(\text {PVLAS-FE })} & =(12 \pm 17) \times 10^{-23} \quad @ B=2.5 \mathrm{~T} \\
\left(\frac{\Delta n}{B_{\text {ext }}^{2}}\right)^{(\text {PVLAS-FE })} & =(19 \pm 27) \times 10^{-24} \mathrm{~T}^{-2}
\end{aligned}
$$

to be compared with the predicted value of $3 A_{e}=4 \times 10^{-24} \mathrm{~T}^{-2}$. These results were obtained by rotating each magnet at a slightly different frequency to keep systematic errors under control and the total composite integration time was $\mathcal{T}=5 \times 10^{6} \mathrm{~s}$.

In Figure 17 the time evolution of different experiments dedicated to measuring vacuum magnetic birefringence is reported. Given the log scale and to take into account the sign of the $\Delta n$ measurements, red markers indicate a birefringence whose central value is 
$\Delta n>0$, black markers indicate $\Delta n<0$ and blue markers indicate values for which the sign of $\Delta n$ was not determined. The error bars correspond to a $1 \sigma$ statistical uncertainty. It is interesting to note how many of the experimental values, reported as limits, are actually not compatible with zero within the declared uncertainty indicating the presence of a systematic contribution.

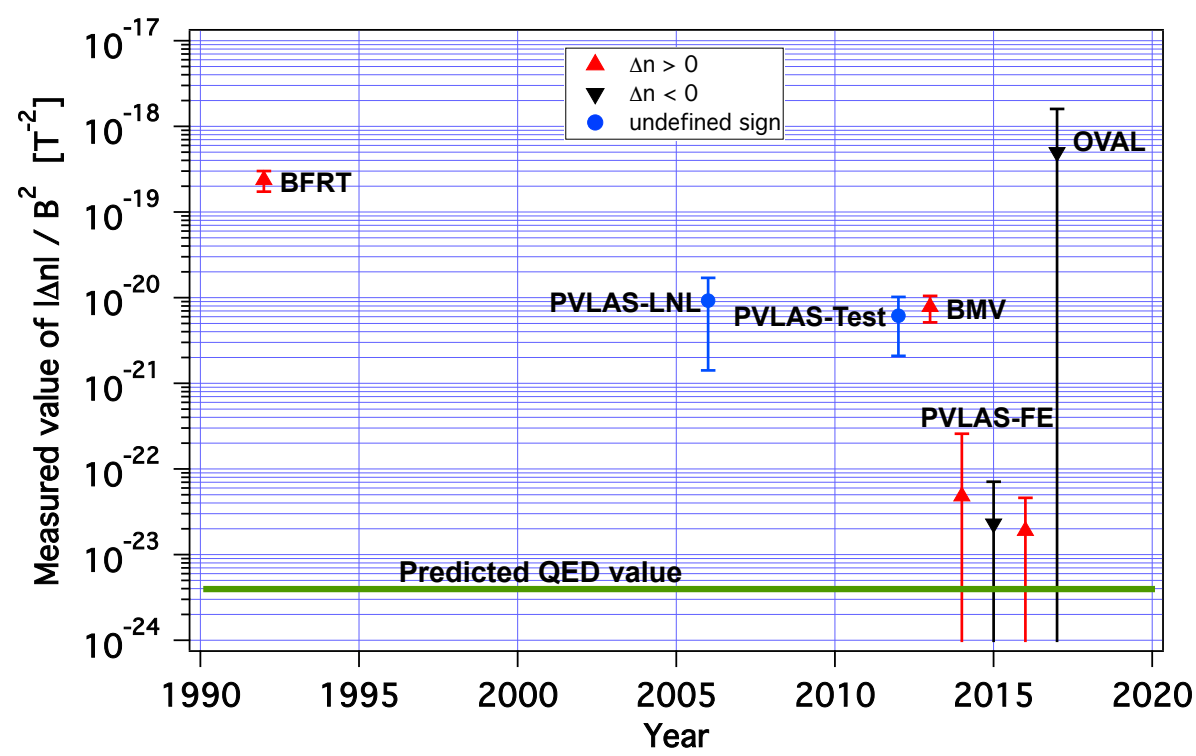

Figure 17. Historical time evolution of the measurement of vacuum magnetic birefringence normalized to $B_{\text {ext }}^{2}$. Error bars correspond to one $\sigma$. The values derive from the following references: BFRT [40]; PVLAS-LNL [44,45], PVLAS-Test [61], BMV [62], PVLAS-FE [59,67], OVAL [63].

The statistical uncertainty of the PVLAS-FE result reported in Figure 17 and in Equation (64), rewritten here for easier reading, is about a factor 7 greater than the expected QED effect obtained with a total integration time of $\mathcal{T}=5 \times 10^{6} \mathrm{~s}$ :

$$
\left(\frac{\Delta n}{B_{\text {ext }}^{2}}\right)^{(\text {PVLAS-FE })}=(19 \pm 27) \times 10^{-24} \mathrm{~T}^{-2} .
$$

\subsection{VMD Measurements}

A vacuum magnetic dichroism limit was also obtained, assuming that a rotation is proportional to the magnetic field length [assumption not true for ALPs as can be deduced from Equations (33) and (26)], leading to

$$
|\Delta \kappa|^{(\mathrm{PVLAS}-\mathrm{FE})}=(10 \pm 28) \times 10^{-23} @ B=2.5 \mathrm{~T} .
$$

However, in the view of setting limits on ALPs coupling constant $g_{a}$ as a function of $m_{a}$ we also report the dichroism limit obtained with both magnets rotating at the same frequency and in phase in a time $T=7 \times 10^{5} \mathrm{~s}$

$$
|\Delta \kappa|^{(\text {PVLAS-FE, } 2 \text { magnets })}=(3 \pm 60) \times 10^{-23} \quad @ B=2.5 \mathrm{~T} .
$$

Finally, given that the coupling constant $g_{a}$ in the low mass limit is related to the dichroism by the relation ${ }^{2}$

$$
g_{a}=\sqrt{\frac{\omega}{2} \frac{\Delta \kappa}{L_{B}}} \frac{4}{B_{\mathrm{ext}}}
$$


in which the coupling constant $g_{a}$ is proportional $\Delta \kappa / L_{B}$, we also report the weighted average of $\Delta \kappa / L_{B}$ for all the PVLAS-FE data (see Reference [1] for details):

$$
\left\langle\frac{\Delta \kappa}{L_{B}}\right\rangle=(1.0 \pm 2.6) \times 10^{-22} \mathrm{~m}^{-1}
$$

where $L_{B}=0.82 \mathrm{~m}$ corresponding to the length of one magnet.

\subsection{Limits on ALP and MCP Searches with PVLAS-FE}

The birefringence and dichroism limits in Equations (64) and (67) can be used to set laboratory limits on the existence of ALPs. Figure 18 is a summary of the existing experimental laboratory limits on the coupling constant $g_{a}$ as a function of the ALP mass $m_{a}$. The shaded regions in the mass-coupling constant plot are excluded. For the dichroism only the two-magnet configuration is plotted to cover the mass range up to $m_{a}=0.1 \mathrm{eV}$.

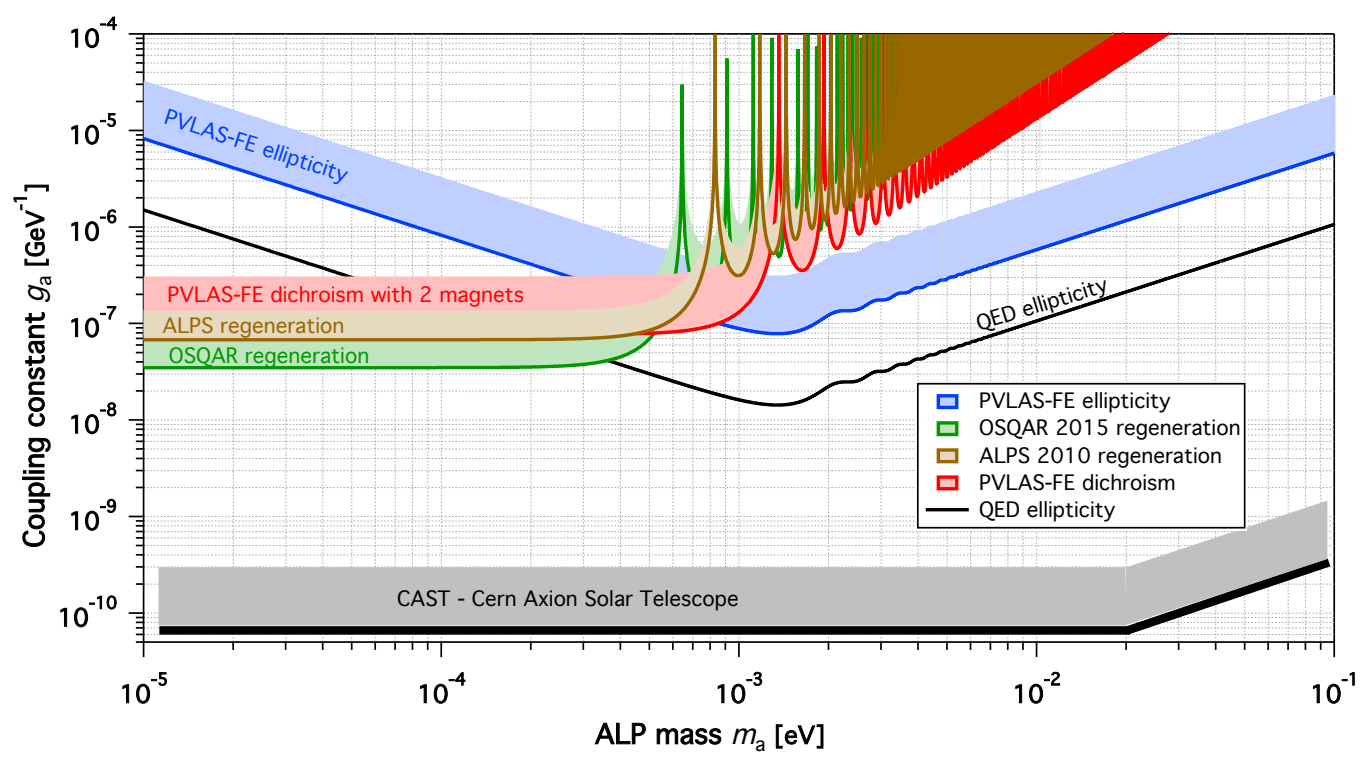

Figure 18. Laboratory limits on the existence of ALPs particles at 95\% c.l. The shaded regions of the graph are excluded. Superimposed on the plot is the ellipticity corresponding to effect of QED vacuum magnetic birefringence. The figure also shows the measurements by the OSQAR [51] and the ALPS [50] collaborations. For comparison, the latest limits by CAST [86] are also shown.

As discussed above a slightly better low mass limit for $m_{a} \ll \sqrt{4 \omega / L_{B}} \approx 1 \mathrm{meV}$ [see Equation (25)] can be set considering Equation (68) and the averaged value of $\Delta \kappa / L_{B}$ in expression (69):

$$
g_{a}^{(95 \%)}<6.4 \times 10^{-8} \mathrm{GeV}^{-1}
$$

at $95 \%$ c.l. As a comparison in the same plot, we report the latest result by the CAST collaboration [86] which uses the Sun as an ALP source. An LHC prototype dipole magnet is aimed at the Sun and X-ray detectors search for regenerated photons. As can be seen, at present laboratory measurements cannot compete with such an experiment.

Finally, from the birefringence and dichroism results in Equations (64) and (66) the exclusion plots shown in Figure 19 for the existence of either Bosonic or Fermionic MCPs can be set. 

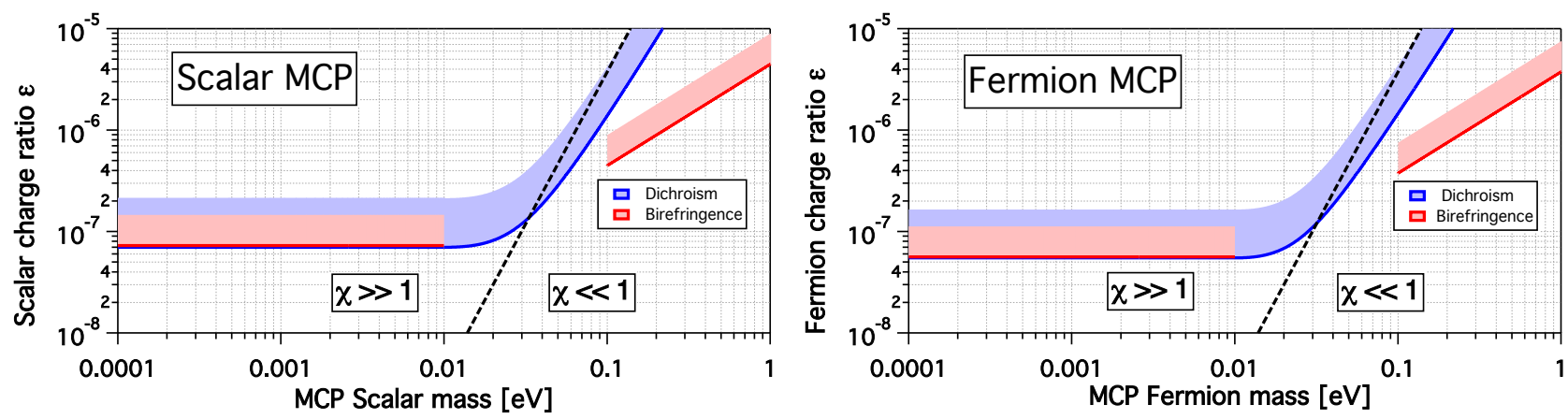

Figure 19. Laboratory exclusion plots based on equations in Section 1.1.2 for the existence of MCPs at 95\% c.1. using the birefringence (red) and dichroism (blue) limits in Equations (64) and (66). The shaded regions of the graph are excluded. The two branches of the birefringence curve are not connected in the mass range around $\chi=1$ (dashed line), where $\Delta n$ changes sign.

\subsection{Future}

Overcoming the factor 7 gap between the measured PVLAS-FE vacuum magnetic birefringence noise floor and the predicted value was unfeasible. To beat the intrinsic noise in optical path difference due to the cavity, shown in Figures 7 and 16, an increase in the VMB optical path difference $\Delta \mathcal{D}$ is necessary:

$$
\Delta \mathcal{D}=3 A_{e} \int_{L_{B}} B_{\mathrm{ext}}^{2} d L
$$

Following Equation (59) a Letter of Intent [3] proposing a new experiment called VMB@CERN has been submitted to CERN to exploit a spare LHC dipole magnet with $\int_{L_{B}} B_{\text {ext }}^{2} d L \approx 1200 \mathrm{~T}^{2} \mathrm{~m}$ and a new polarization modulation polarimetric scheme. With such a magnet and assuming an integration time of order $\mathcal{T} \sim 1$ day to reach SNR $=1$ the necessary sensitivity in optical path difference must be

$$
S_{\Delta \mathcal{D}}^{(\text {desired })} \lesssim 3 A_{e} \int_{L_{B}} B_{\text {ext }}^{2} d L \sqrt{\mathcal{T}} \approx 10^{-18} \mathrm{~m} / \sqrt{\mathrm{Hz}}
$$

Considering the intrinsic cavity mirror noise as the dominant noise such a sensitivity is reached above a few hertz. Assuming an amplification factor $N$ of the Fabry-Perot the necessary sensitivity in ellipticity is $S_{\Psi}^{\text {(desired) }}=\frac{N \pi}{\lambda} S_{\Delta \mathcal{D}}^{\text {(desired) }}$ [see expression (57)]. Furthermore, the ultimate peak ellipticity noise is given by shot-noise and has already been reported in expression (50) which for $\eta_{0} \gg \sigma^{2}, \gamma_{\mathrm{DC}}^{2}$, leads to

$$
S_{\Psi}^{(\text {shot })}=\sqrt{\frac{e}{I_{\|} q}}
$$

independent of $N$. Therefore, by imposing $S_{\Psi}^{(\text {shot })} \lesssim S_{\Psi}^{(\text {desired })}$ a condition on $N$ and $I_{\|}$can be set to design a polarimeter to measure VMB with an LHC dipole magnet:

$$
N \sqrt{I_{\|}}>S_{\Delta \mathcal{D}}^{\text {(desired) }} \frac{\lambda}{\pi} \sqrt{\frac{e}{q}} .
$$

What is being proposed in VMB@CERN is to rotate the polarization inside the static magnetic field instead of varying the magnetic field. The new polarization modulation scheme described in [2,3] is shown in Figure 20. It is based on two co-rotating half-wave plates $\mathrm{L}_{1}$ and $\mathrm{L}_{2}$ inside the cavity, one before the magnetic field and the second after. This choice was necessary to keep the polarization direction fixed on the two Fabry-Perot mirrors. There are two fundamental reasons for this: a rotating polarization on the 
Fabry-Perot mirrors would induce an ellipticity due the intrinsic birefringence of the mirrors themselves appearing at the same frequency as a VMB signal but many orders of magnitude larger; the second reason is that in this configuration only the VMB signal is shifted upwards in frequency whereas the mirror intrinsic noise is not.

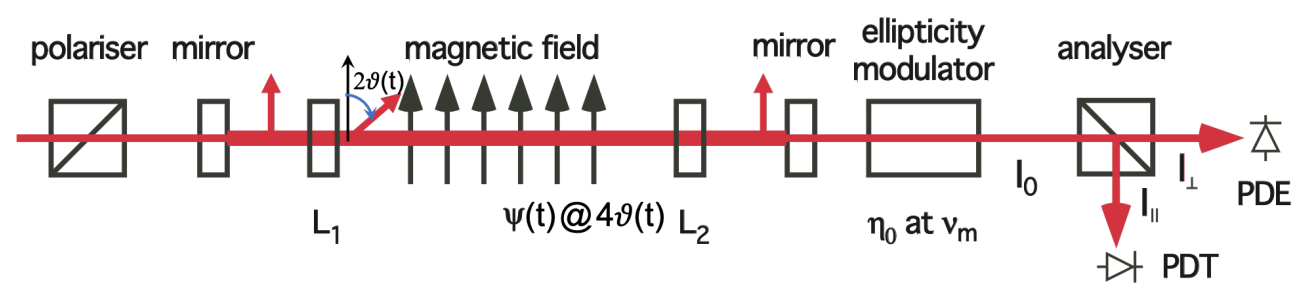

Figure 20. Schematic drawing of the polarization modulation scheme proposed for the VMB@CERN experiment. $\mathrm{L}_{1}$ and $\mathrm{L}_{2}$ are two HWPs co-rotating at $\vartheta(t)$ resulting in a polarization rotation at $2 \vartheta(t)$ only inside the magnetic field. The induced ellipticity will be at $4 \vartheta(t)$. The rest of the scheme is identical to the PVLAS scheme.

The introduction of optical elements inside the Fabry-Perot cavity will introduce losses $p$ such that $R+T+p=1$ and $N=2 /(T+p)$. Commercial anti-reflective coatings have $A R \approx 0.1 \%$ per surface resulting in $p \approx 0.4 \%$. Assuming a dedicated anti-reflective coating a factor 3 better and mirrors with $T \approx 0.05 \%$ then $N \approx 1000$. With an output intensity $I_{\|}=26 \mathrm{~mW}$ the condition in (71) is satisfied $(\lambda=1064 \mathrm{~nm}, q=0.7 \mathrm{~A} / \mathrm{W})$.

Tests are underway in the Ferrara laboratory to study systematics due to the rotating elements. In particular, effects due to non-perfect HWPs (both in retardation and mechanical construction) and non-perfect co-rotation were observed and are presently under study. Solutions have been found and we hope to have a final design working by the end of 2021.

Author Contributions: The two authors gave equal contribution to the paper. All authors have read and agreed to the published version of the manuscript.

Funding: The PVLAS experiment was funded by the Istituto Nazionale di Fisica Nucleare (INFN), Italy, and by Ministero dell'Istruzione, dell'Università e della Ricerca (MIUR), Italy.

Conflicts of Interest: The authors declare no conflict of interest.

\section{Notes}

1 In natural Heaviside-Lorentz units $1 \mathrm{~T}=\sqrt{\frac{\hbar^{3} c^{3}}{e^{4} \mu_{0}}}=195 \mathrm{eV}^{2}$ and $1 \mathrm{~m}=\frac{e}{\hbar c}=5.06 \times 10^{6} \mathrm{eV}^{-1}$.

$2 \quad 1 \mathrm{~T}=\sqrt{\frac{\hbar^{3} c^{3}}{e^{4} \mu_{0}}}=195 \mathrm{eV}^{2}$ and $1 \mathrm{~m}=\frac{e}{\hbar c}=5.06 \times 10^{6} \mathrm{eV}^{-1}$.

\section{References}

1. Ejlli, A.; Della Valle, F.; Gastaldi, U.; Messineo, G.; Pengo, R.; Ruoso, G.; Zavattini, G. The PVLAS experiment: A 25 year effort to measure vacuum magnetic birefringence. Phys. Rep. 2020, 871, 1-74. [CrossRef]

2. Zavattini, G.; Della Valle, F.; Ejlli, A.; Ruoso, G. A polarisation modulation scheme for measuring vacuum magnetic birefringence with static fields. Eur. Phys. C 2016, 76, 294-1-294-5. [CrossRef]

3. Ballou, R.; Kunc, Š.; Pengo, R.; Ruoso, G.; Gastaldi, U.; Milotti, E.; Pugnat, P.; Ejlli, A.; Siemko, A.; Zavattini, G.; et al. Letter of Intent to Measure Vacuum Magnetic Birefringence: The VMB@CERN Experiment; Tech. Rep. CERN-SPSC-2018-036, SPSC-I-249; CERN: Geneva, Switzerland, 2018.

4. $\quad$ Euler, H.; Kockel, B. Über die Streuung von Licht an Licht nach der Diracschen Theorie. Naturwissenschaften 1935, $23,246-247$. [CrossRef]

5. Euler, H. Über die Streuung von Licht an Licht nach der Diracschen Theorie. Ann. Phys. 1936, 26, 398-448. [CrossRef]

6. Heisenberg, W.; Euler, H. Folgerungen aus der Diracschen Theorie des Positrons. Z. Phys. 1936, 98, 714-732. [CrossRef]

7. Weisskopf, V. Über die Elektrodynamik des Vakuums auf Grund der Quantentheorie des Elektrons. Kong. Dan. Vid. Sel. Mat. Fys. Medd. 1936, 14, 1-39.

8. Karplus, R.; Neuman, M. Non-linear interactions between electromagnetic fields. Phys. Rev. 1950, 80, 380-385. [CrossRef]

9. Schwinger, J.S. On gauge invariance and vacuum polarization. Phys. Rev. 1951, 82, 664-679. [CrossRef]

10. Karplus, R.; Neuman, M. The scattering of Light by Light. Phys. Rev. 1951, 83, 776-784. [CrossRef] 
11. Toll, J.S. The Dispersion Relation for Light and Its Applications to Problems Involving Electron Pairs. Ph.D. Thesis, Princeton University, Princeton, NJ, USA, 1952.

12. Erber, T. Velocity of Light in a Magnetic Field. Nature 1961, 190, 25-27. [CrossRef]

13. Klein, J.J.; Nigam, B.P. Birefringence of the vacuum. Phys. Rev. 1964, 135, B1279-B1280. [CrossRef]

14. Baier, R.; Breitenlohner, P. The Vacuum Refraction Index in the Presence of External Fields. Nuovo Cimento 1967, B47, 117-120. [CrossRef]

15. Bialynicka-Birula, Z.; Bialynicki-Birula, I. Nonlinear Effects in Quantum Electrodynamics. Photon Propagation and Photon Splitting in External Fields. Phys. Rev. 1970, D2, 2341-2345. [CrossRef]

16. Adler, S.L. Photon Splitting and Photon Dispersion in a Strong Magnetic Field. Ann. Phys. N. Y. 1971, 67, 599-647. [CrossRef]

17. Aad, G.; Abbott, B.; Abbott, D.C.; Abud, A.A.; Abeling, K.; Abhayasinghe, D.K.; Abidi, S.H.; AbouZeid, O.S.; Abraham, N.L.; Abramowicz, H.; et al. Observation of Light-by-Light Scattering in Ultraperipheral $\mathrm{Pb}+\mathrm{Pb}$ Collisions with the ATLAS Detector. Phys. Rev. Lett. 2019, 123, 052001-1-052001-21. [CrossRef]

18. Mignani, R.P.; Testa, V.; Caniulef, D.G.; Taverna, R.; Turolla, R.; Zane, S.; Wu, K. Evidence for vacuum birefringence from the first optical-polarimetry measurement of the isolated neutron star RXJ1856.5-3754. Mon. Not. R. Astron. Soc. 2016, 465, 492-500. [CrossRef]

19. Capparelli, L.M.; Damiano, A.; Maiani, L.; Polosa, A.D. A note on polarized light from magnetars. Eur. Phys. J. C 2017, 77, 754-1-754-6. [CrossRef]

20. Born, M. Modified Field Equations with a Finite Radius of the Electron. Nature 1933, 132, 282. [CrossRef]

21. Born, M.; Infeld, L. Foundations of the New Field Theory. Nature 1933, 132, 1004. [CrossRef]

22. Born, M.; Infeld, L. Foundations of the New Field Theory. Proc. R. Soc. Lond. Ser. A Math. Phys. Eng. Sci. 1934, $144,425-451$. [CrossRef]

23. Ritus, V.I. Lagrangian of an intensive electromagnetic field and quantum electrodynamics at short distances. Sov. Phys. JETP 1976, 42, 774-782.

24. Gies, H.; Jaeckel, J.; Ringwald, A. Polarized light propagating in a magnetic field as a probe of millicharged fermions. Phys. Rev. Lett. 2006, 97 140402-1-140402-4. [CrossRef]

25. Ahlers, M.; Gies, H.; Jaeckel, J.; Ringwald, A. On the particle interpretation of the PVLAS data: Neutral versus charged particles. Phys. Rev. D 2007, 75, 035011-1-035011-13. [CrossRef]

26. Tsai, W.-Y.; Erber, T. The propagation of photons in homogeneous magnetic fields: Index of refraction. Phys. Rev. D 1975, 12, 1132-1137. [CrossRef]

27. Tsai, W.-Y.; Erber, T. Photon pair creation in intense magnetic fields. Phys. Rev. D 1974, 10, 492-499. [CrossRef]

28. Primakoff, H. Photo-production of neutral mesons in nuclear electric fields and the mean life of the neutral meson. Phys. Rev. 1951, 81, 899. [CrossRef]

29. Peccei, R.D.; Quinn, H.R. CP Conservation in the Presence of Pseudoparticles. Phys. Rev. Lett. 1977, 38, 1440-1443. [CrossRef]

30. Peccei, R.D.; Quinn, H.R. Constraints imposed by CP conservation in the presence of pseudoparticles. Phys. Rev. D 1977, 16, 1791-1797. [CrossRef]

31. Weinberg, S. A New Light Boson? Phys. Rev. Lett. 1978, 40, 223-226. [CrossRef]

32. Wilczek, F. Problem of Strong $P$ and T Invariance in the Presence of Instantons. Phys. Rev. Lett. 1978, 40, 279-282. [CrossRef]

33. Ringwald, A.; Rosenberg, L.J.; Rybka, G. Axions and Other Similar Particles. Prog. Theor. Exp. Phys. 2020, 2020, $939-952$.

34. Graham, P.W.; Irastorza, I.G.; Lamoreaux, S.K.; Lindner, A.; van Bibber, K.A. Experimental Searches for the Axion and Axion-Like Particles. Ann. Rev. Nucl. Part. Sci. 2015, 65, 485-514. [CrossRef]

35. Dine, M.; Fischler, W.; Srednicki, M. A simple solution to the strong CP problem with a harmless axion. Phys. Lett. B 1981, 104, 199-202. [CrossRef]

36. Zhitnitsky, A.R. On Possible Suppression of the Axion Hadron Interactions. Sov. J. Nucl. Phys. 1980, $31,260$.

37. Kuster, M.; Raffelt, G.; Beltrán, B. (Eds.) Axions: Theory, Cosmology, and Experimental Searches; Springer: Berlin/Heidelberg, Germany, 2008.

38. Irastorza, I.G.; Redondo, J. New experimental approaches in the search for axion-like particles. Prog. Part. Nucl. Phys. 2018, 102, 89-159. [CrossRef]

39. Maiani, L.; Petronzio, R.; Zavattini, E. Effects of nearly massless, spin-zero particles on light propagation in a magnetic field. Phys. Lett. B 1986, 175, 359-363. [CrossRef]

40. Cameron, R.; Cantatore, G.; Melissinos, A.; Ruoso, G.; Semertzidis, Y.; Halama, H.J.; Lazarus, D.M.; Prodell, A.G.; Nezrick, F.; Rizzo, C.; et al. Search for nearly massless, weakly coupled particles by optical techniques. Phys. Rev. D 1993, 47, $3707-3725$. [CrossRef] [PubMed]

41. Sikivie, P. Experimental tests of the invisible axion. Phys. Rev. Lett. 1983, 51, 1415-1417; Erratum in 1984, 52, 695. [CrossRef]

42. Raffelt, G.; Stodolsky, L. Mixing of the photon with low-mass particles. Phys. Rev. D 1988, 37, 1237-1249. [CrossRef]

43. Bakalov, D.; Brandi, F.; Cantatore, G.; Carugno, G.; Carusotto, S.; Della Valle, F.; De Riva, A.; Gastaldi, U.; Iacopini, E.; Micossi, P.; et al. The measurement of vacuum polarization: The PVLAS experiment. Hyperfine Interact. 1998, 114, 103-113. [CrossRef]

44. Zavattini, E.; Zavattini, G.; Ruoso, G.; Raiteri, G.; Polacco, E.; Milotti, E.; Lozza, V.; Karuza, M.; Gastaldi, U.; Di Domenico, G.; et al. New PVLAS results and limits on magnetically induced optical rotation and ellipticity in vacuum. Phys. Rev. D 2008, 77, 032006-1-032006-12. [CrossRef] 
45. Bregant, M.; Cantatore, G.; Carusotto, S.; Cimino, R.; Della Valle, F.; Di Domenico, G.; Gastaldi, U.; Karuza, M.; Lozza, V.; Milotti, E.; et al. Limits on low energy photon-photon scattering from an experiment on magnetic vacuum birefringence. Phys. Rev. $D$ 2008, 78, 032006-1-032006-10. [CrossRef]

46. Van Bibber, K.; Dagdeviren, N.R.; Koonin, S.E.; Kerman, A.K.; Nelson, H.N. Proposed experiment to produce and detect light pseudoscalars. Phys. Rev. Lett. 1987, 59, 759-762. [CrossRef] [PubMed]

47. Hoogeveen, F.; Ziegenhagen, T. Production and detection of light bosons using optical resonators. Nucl. Phys. B 1991, 358, 3-26. [CrossRef]

48. Sikivie, P.; Tanner, D.B.; Van Bibber, K. Resonantly Enhanced Axion-Photon Regeneration. Phys. Rev. Lett. 2007, 98, 172002-1-172002-4. [CrossRef]

49. Ruoso, G.; Cameron, R.; Cantatore, G.; Melissinos, A.; Semertzidis, Y.; Halama, H.J.; Lazarus, D.M.; Prodell, A.G.; Nezrick, F.; Rizzo, C.; et al. Search for photon regeneration in a magnetic field. Z. Phys. C 1992, 56, 505-508. [CrossRef]

50. Ehret, K.; Frede, M.; Ghazaryan, S.; Hildebrandt, M.; Knabbe, E.-A.; Kracht, D.; Lindner, A.; List, J.; Meier, T.; Meyer, N.; et al. New ALPS results on hidden-sector lightweights. Phys. Lett. B 2010, 689, 149-155. [CrossRef]

51. Ballou, R.; Deferne, G.; Finger, M. Jr.; Finger, M.; Flekova, L.; Hosek, J.; Kunc, S.; Macuchova, K.; Meissner, K.A.; Pugnat, P.; et al. New exclusion limits on scalar and pseudoscalar axionlike particles from light shining through a wall. Phys. Rev. D 2015, 92, 092002-1-092002-6. [CrossRef]

52. Iacopini, E.; Zavattini, E. Experimental method to detect the vacuum birefringence induced by a magnetic field. Phys. Lett. $B$ 1979, 85, 151-154. [CrossRef]

53. Born, M.; Wolf, E. Principles of Optics, 6th ed.; Pergamon Press: Oxford, UK, 1989.

54. Cantatore, G.; Della Valle, F.; Milotti, E.; Zavattini, E.; Polacco, E.; Perrone, F.; Rizzo, C.; Zavattini, G.; Ruoso, G. Frequency locking of a Nd:YAG laser using the laser itself as the optical phase modulator. Rev. Sci. Instrum. 1995, 66, 2785-2787. [CrossRef]

55. De Riva, A.M.; Zavattini, G.; Marigo, S.; Rizzo, C.; Ruoso, G.; Carugno, G.; Onofrio, R.; Carusotto, S.; Papa, M.; Perrone, F.; et al. Very high $Q$ frequency-locked Fabry-Perot cavity. Rev. Sci. Instrum. 1996, 67, 2680-2684. [CrossRef]

56. Bregant, M.; Cantatore, G.; Della Valle, F.; Ruoso, G.; Zavattini, G. Frequency locking to a high-finesse Fabry-Perot cavity of a frequency doubled Nd:YAG laser used as the optical phase modulator. Rev. Sci. Instrum. 2002, 73, 4142-4144. [CrossRef]

57. Brandi, F.; Ruoso, G.; Polacco, E. Stress-optic modulator: A novel device for high sensitivity linear birefringence measurements. Meas. Sci. Technol. 2001, 12, 1503-1508. [CrossRef]

58. Zavattini, G.; Cantatore, G.; Cimino, R.; Di Domenico, G.; Della Valle, F.; Karuza, M.; Milotti, E.; Ruoso, G. On measuring birefringences and dichroisms using Fabry-Pérot cavities. Appl. Phys. B 2006, 83, 571-577. [CrossRef]

59. Della Valle, F.; Ejlli, A.; Gastaldi, U.; Messineo, G.; Milotti, E.; Pengo, R.; Ruoso, G.; Zavattini, G. The PVLAS experiment: measuring vacuum magnetic birefringence and dichroism with a birefringent Fabry-Perot cavity. Eur. Phys. J. C 2016, 76, 24-1-24-15. [CrossRef]

60. Zavattini, G.; Della Valle, F.; Ejlli, E.; Ni, W.-T.; Gastaldi, U.; Milotti, E.; Pengo, R.; Ruoso, G. Intrinsic mirror noise in Fabry-Perot based polarimeters: the case for the measurement of vacuum magnetic birefringence. Eur. Phys. J. C 2018, 78, 585-1-585-16. [CrossRef]

61. Della Valle, F.; Gastaldi, U.; Messineo, G.; Milotti, E.; Pengo, R.; Piemontese, L.; Ruoso, G.; Zavattini, G. Measurements of vacuum magnetic birefringence using permanent dipole magnets: the PVLAS experiment. New J. Phys. 2013, 15, 053026-1-053026-24. [CrossRef]

62. Cadène, A.; Berceau, P.; Fouché, M.; Battesti, R.; Rizzo, C. Vacuum magnetic linear birefringence using pulsed fields: status of the BMV experiment. Eur. Phys. J. D 2014, 68, 16-1-16-7. [CrossRef]

63. Fan, X.; Kamioka, S.; Inada, T.; Yamazaki, T.; Namba, T.; Asai, S.; Omachi, J.; Yoshioka, K.; Kuwata-Gonokami, M.; Matsuo, A.; et al. The OVAL experiment: a new experiment to measure vacuum magnetic birefringence using high repetition pulsed magnets. Eur. Phys. J. D 2017, 71, 308-1-308-10. [CrossRef]

64. Rizzo, C.; Rizzo, A.; Bishop, D.M. The Cotton-Mouton effect in gases: experiment and theory. Int. Rev. Phys. Chem. 1997, 16, 81-111. [CrossRef]

65. Bregant, M.; Cantatore, G.; Carusotto, S.; Cimino, R.; Della Valle, F.; Di Domenico, G.; Gastaldi, U.; Karuza, M.; Lozza, V.; Milotti, E.; et al. New precise measurement of the Cotton-Mouton effect in helium. Chem. Phys. Lett. 2009, 471, 322-325. [CrossRef]

66. Cadène, A.; Sordes, D.; Berceau, P.; Fouché, M.; Battesti, R.; Rizzo, C. Faraday and Cotton-Mouton effects of helium at $\lambda=1064 \mathrm{~nm}$. Phys. Rev. A 2013, 88, 043815-1-043815-11. [CrossRef]

67. Della Valle, F.; Milotti, E.; Ejlli, A.; Messineo, G.; Piemontese, L.; Zavattini, G.; Gastaldi, U.; Pengo, R.; Ruoso, G. First results from the new PVLAS apparatus: A new limit on vacuum magnetic birefringence. Phys. Rev. D 2014, 90, 092003-1-092003-7. [CrossRef]

68. Bregant, M.; Cantatore, G.; Carusotto, S.; Cimino, R.; Della Valle, F.; Di Domenico, G.; Gastaldi, U.; Karuza, M.; Milotti, E.; Polacco, E.; et al. A precise measurement of the Cotton-Mouton effect in neon. Chem. Phys. Lett. 2005, 410, 288-292. [CrossRef]

69. Bregant, M.; Cantatore, G.; Carusotto, S.; Cimino, R.; Della Valle, F.; Di Domenico, G.; Gastaldi, U.; Karuza, M.; Lozza, V.; Milotti, E.; et al. Erratum to 'Measurement of the Cotton-Mouton effect in krypton and xenon at 1064 nm with the PVLAS apparatus' [Chem. Phys. Lett. 392 (2004) 276] and 'A precise measurement of the Cotton-Mouton effect in neon' [Chem. Phys. Lett. 410 (2005) 288]. Chem. Phys. Lett. 2009, 477, 415. [CrossRef] 
70. Mei, H.H.; Ni, W.T.; Chen, S.J.; Pan, S.S. Measurement of the Cotton-Mouton effect in nitrogen, oxygen, carbon dioxide, argon, and krypton with the Q \& A apparatus. Chem. Phys. Lett. 2009, 471, 216-221.

71. Della Valle, F.; Ejlli, A.; Gastaldi, U.; Messineo, G.; Milotti, E.; Pengo, R.; Piemontese, L.; Ruoso, G.; Zavattini, G. Measurement of the Cotton Mouton effect of water vapour. Chem. Phys. Lett. 2014, 592, 288-291. [CrossRef]

72. Lukins, P.B.; Ritchie, G.L.D. Vapor-phase Cotton-Mouton effects of methane, methyl chloride, chloroform, and carbon tetrachloride. J. Phys. Chem. 1988, 92, 2013-2015. [CrossRef]

73. Bregant, M.; Cantatore, G.; Carusotto, S.; Cimino, R.; Della Valle, F.; Di Domenico, G.; Gastaldi, U.; Karuza, M.; Milotti, E.; Polacco, E.; et al. Measurement of the Cotton-Mouton effect in krypton and xenon at $1064 \mathrm{~nm}$ with the PVLAS apparatus. Chem. Phys. Lett. 2004, 392, 276-280. [CrossRef]

74. Cadene, A.; Fouché, M.; Rivere, A.; Battesti, R.; Coriani, S.; Rizzo, A.; Rizzo, C. Circular and linear magnetic birefringences in xenon at $\lambda=1064 \mathrm{~nm}$. J. Chem. Phys. 2015, 142, 124313-1-124313-10. [CrossRef]

75. Chen, S.-J.; Mei, H.-H.; Ni, W.-T. Q \& A experiment to search for vacuum dichroism, pseudoscalar-photon interaction and millicharged fermions. Mod. Phys. Lett. A 2007, 22, 2815-2831.

76. Brandi, F.; Della Valle, F.; Micossi, P.; De Riva, A.M.; Zavattini, G.; Perrone, F.; Rizzo, C.; Ruoso, G. Cotton-Mouton effect of molecular oxygen: A novel measurement. J. Opt. Soc. Am. B 1998, 15, 1278-1281. [CrossRef]

77. Superconducting Magnet Record. CERN Bull. 1982, 28, 1.

78. Iacopini, E.; Lazeyras, P.; Smith, B.; Picasso, E.; Polacco, E.; Morpuirgo, M.; Zavattini, E. Experimental Determination of Vacuum Polarization Effects on a Laser Light-Beam Propagating in a Strong Magnetic Field; Tech. Rep. Proposal D2; CERN: Geneva, Switzerland, 1980.

79. Carusotto, S.; Scuri, F.; Smith, B.; Iacopini, E.; Picasso, E.; Polacco, E.; Lazeyras, P.; Stefanini, F.; Morpuirgo, M.; Zavattini, E. Addendum to the Proposal D2: Experimental Determination of Vacuum Polarization Effects on a Laser Light-Beam Propagating in a Strong Magnetic Field; Tech. Rep. Proposal D2-Add; CERN: Geneva, Switzerland, 1983.

80. Multilams First Choice in Scientific Experiments. MC ${ }^{\circledR} /$ HCK $^{\circledR}$ News. 13 March 1999. Available online: https://ec.staubli.com/ AcroFiles/Catalogues/News / Archiv / Archiv/SZ_News-1999_(en)_hi.pdf (accessed on 31 May 2020)

81. Pengo, R.; Petrucci, G.; Marigo, S. An original rotating cryostat for the experiment PVLAS. In Proceedings of the 17th International Cryogenic Engineering Conference (ICEC 17), Bournemouth, UK, 14-17 July 1998; Dew-Hughes, D., Scurlock, R.G., Watson, J.H.P., Eds.; IOP: Bristol, UK, 1998; pp. 851-854.

82. Claudet, G.; Lacaze, A.; Roubeau, P.; Verdier, J. The design and operation of a refrigerator system using superfluid helium. In Proceedings of the 5th International Cryogenic Engineering Conference (ICEC 5), Kyoto, Japan, 7-10 May 1974; Mendelssohn, K., Ed.; IPC Science and Technology Press: Guildford, UK, 1974; pp. 265-267.

83. Bakalov, D.; Brandi, F.; Cantatore, G.; Carugno, G.; Carusotto, S.; Della Valle, F.; De Riva, A.M.; Gastaldi, U.; Iacopini, E.; Micossi, P.; et al. Experimental method to detect the magnetic birefringence of vacuum. Quantum Semiclass. Opt. 1998, 10, 239-250. [CrossRef]

84. Della Valle, F.; Di Domenico, G.; Gastaldi, U.; Milotti, E.; Pengo, R.; Ruoso, G.; Zavattini, G. Towards a direct measurement of vacuum magnetic birefringence: PVLAS achievements. Opt. Commun. 2010, 283, 4194-4198. [CrossRef]

85. Halbach, K. Design of permanent multipole magnets with oriented rare earth cobalt material. Nucl. Instrum. Methods 1980, 169, 1-10. [CrossRef]

86. CAST Collaboration. New CAST limit on the axion-photon interaction. Nat. Phys. 2017, 13, 584-590. [CrossRef] 